TRANSACTIONS OF THE

AMERICAN MATHEMATICAL SOCIETY

Volume 349, Number 4, April 1997, Pages 1527-1550

S 0002-9947(97)01790-X

\title{
INTERSECTION LAWSON HOMOLOGY
}

\author{
PAWEE GAJER
}

\begin{abstract}
The aim of this paper is to construct and describe basic properties of a theory that unifies Lawson homology and intersection homology. It is shown that this theory has a localization sequence, is functorial, satisfies a property analogous to the Lawson Suspension Theorem, and is equipped with an operation analogous to the Friedlander-Mazur s-operation.
\end{abstract}

Intersection theory plays a key role in the theory of smooth manifolds in both differential and algebro-geometric contexts. The main ingredient of intersection theory is intersection pairing. If $X$ is a smooth orientable manifold of dimension $n$, then intersection pairing is defined on the level of the ordinary homology of $X$

$$
H_{k}(X ; \mathbb{Z}) \times H_{l}(X ; \mathbb{Z}) \longrightarrow H_{k+l-n}(X ; \mathbb{Z}) .
$$

If $X$ is a smooth complex algebraic variety of dimension $n$, then intersection pairing is defined on the quotients of the spaces of algebraic cycles of $X$ by the appropriate equivalence relations. For example, there is a refinement

$$
\mathcal{A}_{k}(X) \times \mathcal{A}_{l}(X) \longrightarrow \mathcal{A}_{k+l-n}(X) .
$$

of the pairing (1), where $\mathcal{A}_{k}(X)$ is the group of algebraic $k$-cycles on $X$ modulo algebraic equivalence.

Friedlander and Gabber showed that the intersection pairing (2) is a special case of a much more general pairing that takes place on the level of spaces of algebraic cycles $[\mathrm{FG}]$. In particular, they showed that for every smooth complex projective variety $X$ of dimension $n$ if $k+l \geq n$, then there is a pairing

$$
Z_{k}(X) \wedge Z_{l}(X) \longrightarrow Z_{k+l-n}(X)
$$

which extends, up to homotopy, the usual intersection pairing on cycles that meet in proper dimension. This pairing is homotopy commutative and associative. As a consequence, we get a pairing

$$
\pi_{r} Z_{k}(X) \times \pi_{s} Z_{l}(X) \longrightarrow \pi_{r+s} Z_{k+l-n}(X),
$$

which for $r=s=0$ is the classical intersection pairing (2). The homotopy groups $\pi_{r} Z_{k}(X)$ of the spaces $Z_{k}(X), k \geq 0$, of algebraic cycles on $X$ are called the Lawson homology of $X$ (for more details on this subject see the survey [L1]).

In the case of singular varieties the situation is much more complicated. It is easy to see that on a generic singular variety neither of the pairings (1), (2) is well defined. In the category of pseudo-manifolds this problem was overcome around 1980 by Goresky and MacPherson who came up with an appropriate analogue of

Received by the editors September 30, 1995.

1991 Mathematics Subject Classification. Primary 14F99, 55N99; Secondary 14C05.

Research at MSRI was supported in part by NSF grant \#DMS 9022140.

(C)1997 American Mathematical Society 
ordinary homology for pseudo-manifolds [GM]. For every stratified space $X$ and every function $\bar{p}: \mathbb{Z}_{+} \rightarrow \mathbb{Z}_{+}$so that

$$
\bar{p}(k) \leq \bar{p}(k+1) \leq \bar{p}(k)+1
$$

they defined the intersection homology groups $I_{\bar{p}} H_{k}(X ; \mathbb{Z})$ of $X$ so that

$$
I_{\bar{p}} H_{k}(X) \cong \begin{cases}H^{\operatorname{dim} X-k}(X) & \text { for } \bar{p}=\overline{0} \\ H_{k}(X) & \text { for } \bar{p}=\bar{t}\end{cases}
$$

where $\overline{0}(k)=0$ for $k \geq 0, \bar{t}(0)=\bar{t}(1)=\bar{t}(2)=0$, and $\bar{t}(k)=k-2$ for $k \geq 2$. The functions $\bar{p}$ are called perversities and they control the excess of intersection of cycles with singular strata. The key property of intersection homology is existence of intersection product

$$
I_{\bar{p}} H_{k}(X) \times I_{\bar{q}} H_{l}(X) \longrightarrow I_{\bar{p}+\bar{q}} H_{(k+l)-n}(X)
$$

on any orientable pseudomanifold $X$ of dimension $n$.

One of the questions Goresky and MacPherson raised in their foundational paper [GM] concerned existence of an analogue of intersection homology for the groups $\mathcal{A}_{*}(X)$. A similar question was asked by Friedlander and Lawson in connection with Lawson homology. The goal of this paper is to describe a candidate for such a theory. We will refer to it as intersection Lawson homology. Although we were unable to construct an intersection pairing for intersection Lawson homology, we prove a localization and complex suspensions results whose ordinary Lawson homology versions, extended to the realm of quasi-projective varieties, were key ingredients in the construction of the Friedlander-Gabber intersection pairing (3). Before we formulate the properties of intersection Lawson homology let us describe the construction of this theory.

Dold and Thom proved that for every polyhedron $X$ there is an isomorphism

$$
\pi_{r}\left(Z_{0}(X)\right) \cong H_{r}(X ; \mathbb{Z}) \text {. }
$$

Thus the ordinary Lawson homology of a variety $X$ corresponds on the level of zero cycles to the ordinary homology of $X$. It is natural to expect that on the level of zero cycles the intersection Lawson homology of $X$ corresponds to the intersection homology of $X$. In $[\mathrm{Gr}]$ we have introduced a notion of intersection homotopy groups and proved the following result.

The Intersection Dold-Thom Theorem. For every perversity $\bar{q}$ and $r \geq 0$ there is an isomorphism

$$
I_{\bar{q}} \pi_{r}\left(Z_{0}(X)\right) \cong I_{\bar{q}} H_{r}(X ; \mathbb{Z})
$$

where $I_{\bar{q}} \pi_{r}\left(Z_{0}(X)\right)$ stands for the intersection homotopy groups of $Z_{0}(X)$, taken with respect to a finite filtration $Z_{0}(X)$ of $Z_{0}(X)$ induced by a stratification $X$ of $X$.

The key idea behind the construction of intersection Lawson homology is to apply intersection homotopy groups to spaces of algebraic cycles equipped with some finite filtrations generalizing the filtration $Z_{0}(X)$ of $Z_{0}(X)$. In the next few paragraphs we outline the construction of intersection homotopy groups, state their basic properties, and describe certain finite filtrations on spaces of algebraic cycles.

If $X$ is a space equipped with a finite filtration

$$
X: X=X^{0} \supset X^{1} \supset X^{2} \supset \cdots \supset X^{n} \supset \emptyset
$$


then the elements of the intersection homotopy group $I_{\bar{q}} \pi_{r}(X)$ are homotopy classes of pointed maps $f: \mathbb{S}_{r} \rightarrow X$ from the unit $r$-dimensional sphere $\mathbb{S}_{r}$ into $X$, where both maps and homotopies satisfy the perversity $\bar{q}$ condition

$$
\operatorname{dim} f^{-1}\left(X^{i}\right) \leq \operatorname{dim}(\operatorname{dom}(f))-i+\bar{q}(i)
$$

with respect to the filtration $X=\left\{X^{i}\right\}$ of $X$, where $\operatorname{dom}(f)$ is the domain of the map $f$. Intersection homotopy groups have many properties analogous to those of ordinary homotopy groups. For example, for every $\bar{q}$, the perversity $\bar{q}$ fundamental group $I_{\bar{q}} \pi_{1}(X)$ acts on $I_{\bar{q}} \pi_{r}(X)$, there is an intersection Hurewicz homomorphism

$$
I_{\bar{q}} \pi_{r}(X) \longrightarrow I_{\bar{q}} H_{r}(X ; \mathbb{Z})
$$

and there are long exact sequences of intersection homotopy groups for filtered fibrations and pairs.

A finite filtration

$$
Z_{0}(X): \quad Z_{0}(X)=Z_{0}(X)^{0} \supset Z_{0}(X)^{1} \supset Z_{0}(X)^{2} \supset \cdots \supset Z_{0}(X)^{n} \supset \emptyset,
$$

of $Z_{0}(X)$ from the Intersection Dold-Thom Theorem is called a Lawson filtration of $Z_{0}(X)$ and it is defined as follows.

$$
\sum n_{i} x_{i} \in Z_{0}(X)^{k}
$$

if there is $n_{i} \neq 0$ so that $x_{i}$ belongs to the codimension $k$ skeleton $X^{k}$ of the stratification $X=\left\{X^{s}\right\}_{s=0}^{n}$. It turns out that for every $k \geq 1$ the space $Z_{k}(X)$ has many filtrations that are natural generalization of the Lawson filtration of $Z_{0}(X)$ and all of them can be parametrized by perversities. Thus, with every stratification $X$ of $X$ and every perversity $\bar{p}$ we can associate a finite filtration

$$
Z_{k}(X)^{\bar{p}}: \quad Z_{k}(X)=Z_{k}(X)^{\bar{p}, 0} \supset Z_{k}(X)^{\bar{p}, 1} \supset Z_{k}(X)^{\bar{p}, 2} \supset \cdots \supset Z_{k}(X)^{\bar{p}, n} \supset \emptyset
$$

of $Z_{k}(X)$ so that for $k=0$ and $\bar{p}=\bar{t}_{c}$ the filtration $Z_{0}(X)^{\bar{t}_{c}}$ is the Lawson filtration of $Z_{0}(X)$, where $\bar{t}_{c}$ is the complex top perversity so that $\bar{t}_{c}(0)=\bar{t}_{c}(1)=0$ and $\bar{t}_{c}(i)=i-1$ for $i \geq 1$. The strata of the filtration $Z_{k}(X)^{\bar{p}}$ have the following structure. The regular stratum $Z_{k}(X)-Z_{k}(X)^{\bar{p}, 1}$ consists of algebraic $k$-cycles $c=\sum n_{i} c_{i}$ of $X$ satisfying for every skeleton $X^{j}$ of a stratification $X=\left\{X^{i}\right\}$ of $X$ the following perversity $\bar{p}$ condition:

$$
\operatorname{dim}\left(|c| \cap X^{j}\right) \leq k-j+\bar{p}(j),
$$

where $|c|$ is the support of the cycle $c$. The formal codimension $i$ stratum $Z_{k}(X)^{\bar{p}, i}-$ $Z_{k}(X)^{\bar{p}, i+1}$ consists of those $k$-cycles of $X$ that violate the perversity $\bar{p}$ condition (4) on the codimension $i$ skeleton $X^{i}$ of $\mathcal{X}$ and satisfies perversity $\bar{p}$ condition on skeleta of $X$ of codimension greater than $i$.

The intersection Lawson homology groups of a variety $X$ are the intersection homotopy groups

$$
I_{\bar{q}} \pi_{r}\left(Z_{k}(X), Z_{k}(X)^{\bar{p}}\right)
$$

of $Z_{k}(X)$ with respect to the perversity $\bar{p}$ filtration $Z_{k}(X)^{\bar{p}}$ of $Z_{k}(X)$. Thus every element of the group $I_{\bar{q}} \pi_{r}\left(Z_{k}(X), Z_{k}(X)^{\bar{p}}\right)$ is represented by a continuous family of algebraic $k$-cycles of $X$

$$
f: \mathbb{S}_{r} \longrightarrow Z_{k}(X)
$$

for which there is a filtration

$$
\mathbb{S}_{r}=V^{0} \supset V^{1} \supset V^{2} \supset \cdots \supset \emptyset
$$


of the sphere $\mathbb{S}_{r}$ by closed subsets so that $\operatorname{dim} V^{i} \leq m-i-\bar{q}(i)$, for every $x \in$ $\mathbb{S}_{r}-V^{1}$ the cycle $f(x)$ satisfies the perversity $\bar{p}$ condition (4) on every skeleton of the stratification $\mathcal{X}$ of $X$, and $f$ restricted to $V^{i}-V^{i+1}$ represents a family of cycles that satisfy the perversity $\bar{p}$ condition on all skeleta of $\mathcal{X}$ of codimension greater than $i$ and violate the perversity $\bar{p}$ condition on the codimension $i$ skeleta of $\mathcal{X}$.

Since there is an isomorphism

$$
I_{\bar{q}} \pi_{r}\left(Z_{0}(X), Z_{0}(X)^{\bar{t}_{c}}\right) \cong I_{\bar{q}} H_{r}(X ; \mathbb{Z})
$$

the groups $I_{\bar{q}} \pi_{r}\left(Z_{0}(X), Z_{0}(X)^{\bar{c}_{c}}\right)$ do not depend of the choice of a stratification $X$ of $X$. Unfortunately, we cannot prove a similar result for spaces of higher dimensional cycles. To ensure that the groups $I_{\bar{q}} \pi_{r}\left(Z_{0}(X), Z_{0}(X)^{\bar{t}_{c}}\right)$ depend only on the isomorphism type of $X$ in the sequel we take $X$ to be the canonical Whitney stratification $X_{c a n}$ of $X$ (for more details on this stratification see Section 1 and $[\operatorname{Tr}])$. By the abuse of notation we will write $I_{\bar{q}} \pi_{r}\left(Z_{k}(X)^{\bar{p}}\right)$ in the place of $I_{\bar{q}} \pi_{r}\left(Z_{k}(X), Z_{k}\left(X_{c a n}\right)^{\bar{p}}\right)$ and we will denote by $Z_{k}(X)^{\bar{p}}$ the canonical perversity $\bar{p}$ filtration $Z_{k}\left(X_{c a n}\right)^{\bar{p}}$ of $Z_{k}(X)$.

For some extreme choices of perversities $\bar{p}, \bar{q}$, and integers $r, k$ the intersection Lawson homology groups $I_{\bar{q}} \pi_{r}\left(Z_{k}(X)^{\bar{p}}\right)$ of $X$ have the following description.

As was mentioned above the groups $I_{\bar{q}} \pi_{r}\left(Z_{0}(X), Z_{0}(X)^{\bar{t}_{c}}\right)$ are isomorphic to the intersection homology groups $I_{\bar{q}} H_{r}(X ; \mathbb{Z})$ of $X$. If $\bar{p}$ and $\bar{q}$ are the identity maps, then $I_{\bar{q}} \pi_{r}\left(Z_{k}(X)^{\bar{p}}\right)$ is isomorphic to the ordinary Lawson homology $\pi_{r}\left(Z_{k}(X)\right)$ of $X$. When $r=0$ and $\bar{q}$ is a perversity so that $\bar{q}(0)=\bar{q}(1)=0$, then there is an isomorphism

$$
I_{\bar{q}} \pi_{0}\left(Z_{k}(X)^{\bar{p}}\right) \cong \pi_{0}\left(I_{\bar{p}} Z_{k}(X)\right),
$$

where $I_{\bar{p}} Z_{k}(X)=Z_{k}(X)-Z_{k}(X)^{\bar{p}, 1}$ is the set of perversity $\bar{p} k$-cycles of $X$. The group $\pi_{0}\left(I_{\bar{p}} Z_{k}(X)\right)$ can be identified with the quotient of $Z_{k}(X)$ by a perversity $\bar{p}$ algebraic equivalence relation (for more details see Section 1). The groups $I_{\bar{p}} Z_{k}(X)$ essentially coincide with Flannery's groups $Z_{k, \bar{p}}(X)$ of $\bar{p}$-allowable $k$-cycles on $X$, which are defined using perversity conditions in terms of real dimensions of intersections of cycles with strata [Fl]. Flannery takes the homotopy groups of $Z_{k, \bar{p}}(X)$ as an another extension of Lawson homology to the singular context.

The following two theorems are the main results of this paper.

Theorem 2.1. If $V \subset X$ is a pair of projective varieties so that $V_{c a n}^{l}=V \cap X_{c a n}^{l}$ for every canonical skeleton $V_{c a n}^{l}$ of $V$, then there is a localization long exact sequence

$\cdots \rightarrow I_{\bar{q}} \pi_{r}\left(Z_{k}(V)^{\bar{p}}\right) \rightarrow I_{\bar{q}} \pi_{r}\left(Z_{k}(X)^{\bar{p}}\right) \rightarrow I_{\bar{q}} \pi_{r}\left(Z_{k}(X, V)^{\bar{p}}\right) \rightarrow I_{\bar{q}} \pi_{r-1}\left(Z_{k}(V)^{\bar{p}}\right) \rightarrow \cdots$

where $Z_{k}(X, V)^{\bar{p}}$ is the image of the canonical perversity $\bar{p}$ filtration $Z_{k}(X)^{\bar{p}}$ of $Z_{k}(X)$ by the projection

$$
Z_{k}(X) \longrightarrow Z_{k}(X, V) \stackrel{\text { def }}{=} Z_{k}(X) / Z_{k}(V) .
$$

Theorem 3.3. If $X$ is a projective variety and $\mathcal{O}_{X}(1) \rightarrow X$ is the hyperplane line bundle on $X \subset \mathbb{P}_{n}$, then for every pair of non-negative integers $k, r$, every perversity $\bar{q}$, and every perversity $\bar{p}$ so that for every $i \geq 0$ there is an inequality $k-i+1+\bar{p}(i) \geq 0$ the projection $\mathcal{O}_{X}(1) \rightarrow X$ induces an isomorphism

$$
I_{\bar{q}} \pi_{r}\left(Z_{k}(X)^{\bar{p}}\right) \longrightarrow I_{\bar{q}} \pi_{r}\left(Z_{k+1}\left(\mathcal{O}_{X}(1)\right)^{\bar{p}}\right) .
$$


The space $Z_{k+1}\left(\mathcal{O}_{X}(1)\right)$ can be identified with $Z_{k+1}(\not X)$, where $\$ X X$ be a complex join of $X$ with a point $x_{0}$ in $\mathbb{P}_{n+1}-\mathbb{P}_{n}$, for some linear embedding $\mathbb{P}_{n} \hookrightarrow \mathbb{P}_{n+1}$. The filtration $Z_{k+1}\left(\mathcal{O}_{X}(1)\right)^{\bar{p}}$ of $Z_{k+1}\left(\mathcal{O}_{X}(1)\right)$ has the following skeleta:

$$
Z_{k+1}\left(\mathcal{O}_{X}(1)\right)^{\bar{p}, 0}=Z_{k+1}\left(\mathcal{O}_{X}(1)\right)
$$

and $c \in Z_{k+1}\left(\mathcal{O}_{X}(1)\right)^{\bar{p}, l}$ for some $l \geq 1$ if there is $j \geq l$ so that

$$
\operatorname{dim}\left(|c| \cap \pi^{-1} X_{c a n}^{j}\right) \geq k-j+\bar{p}(j)+1 .
$$

Theorem 2.1 is a generalization of Lima-Filho's localization long exact sequence

$$
\cdots \longrightarrow \pi_{r}\left(Z_{k}(V)\right) \longrightarrow \pi_{r}\left(Z_{k}(X)\right) \longrightarrow \pi_{r}\left(Z_{k}(X, V)\right) \longrightarrow \pi_{r-1}\left(Z_{k}(V)\right) \longrightarrow \cdots
$$

and Theorem 3.3 generalizes the Lawson Suspension Theorem, which says that for every projective variety $X$, and every pair of non-negative integers $k, r$, there is an isomorphism

$$
\pi_{r}\left(Z_{k}(X)\right) \longrightarrow \pi_{r}\left(Z_{k+1}(\not X)\right)
$$

We also show that Friedlander's and Mazur's s-operation

$$
\mathbf{s}: \pi_{r} Z_{k}(X) \longrightarrow \pi_{r+2} Z_{k-1}(X),
$$

lifts to the intersection Lawson homology of $X$.

Theorem 4.1. Let $X$ be a projective variety. Then for every pair of non-negative integers $k, r$, every perversity $\bar{q}$, and every perversity $\bar{p}$ so that for every $i \geq 0$ we have $k-i+\bar{p}(i) \geq 0$, there is a homomorphism

$$
\mathbf{s}_{\bar{p}, \bar{q}}: I_{\bar{q}} \pi_{r}\left(Z_{k}(X)^{\bar{p}}\right) \longrightarrow I_{\bar{q}} \pi_{r+2}\left(Z_{k-1}(X)^{\bar{p}}\right),
$$

so that the diagram

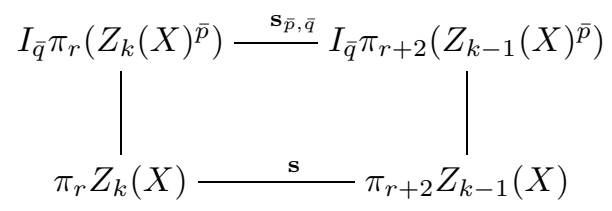

commutes.

As a corollary we get the following result.

Corollary 4.2. The composition of $\mathbf{s}_{\bar{t}_{c}, \bar{q}}$ and $\mathbf{s}$ homomorphisms induces the following commutative diagram:

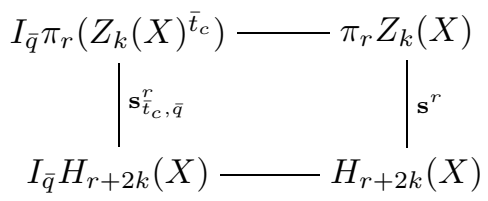

Intersection Lawson homology is functorial with respect to filtered and cofiltered maps. A map $f: X \rightarrow Y$ of complex quasi-projective varieties is called filtered (resp. cofiltered) if for every $l \geq 0$ we have $f^{-1}\left(Y_{\text {can }}^{l}\right) \supset X_{\text {can }}^{l}\left(\operatorname{resp} . f^{-1}\left(Y_{\text {can }}^{l}\right) \subset X_{\text {can }}^{l}\right)$. The examples of both filtered and cofiltered maps include: smooth morphisms, embeddings of Zariski open subsets, embeddings of generic hyperplane sections into a projective variety. 
We show that for every pair of projective varieties $X$ and $Y$ each cofiltered proper morphism $f: X \rightarrow Y$ induces a homomorphism

$$
f_{*}: I_{\bar{q}} \pi_{r}\left(Z_{k}(X)^{\bar{p}}\right) \longrightarrow I_{\bar{q}} \pi_{r}\left(Z_{k}(Y)^{\bar{p}}\right)
$$

and each filtered flat morphism $f: X \rightarrow Y$ of relative dimension $s$ induces a homomorphism

$$
f^{*}: I_{\bar{q}} \pi_{r}\left(Z_{k}(Y)^{\bar{p}}\right) \longrightarrow I_{\bar{q}} \pi_{r}\left(Z_{k+s}(X)^{\bar{p}}\right) .
$$

The paper is organized as follows. In Section 1 we define perversity $\bar{p}$ Lawson filtrations, recall a definition of intersection homotopy groups and their basic properties, define intersection Lawson homology, study its functoriality properties, and discuss some examples. In particular, we describe intersection homology analogues of the groups $\mathcal{A}_{*}(X)$ of algebraic equivalence classes of algebraic cycles of $X$. Section 2 is devoted to localization long exact sequence for intersection Lawson homology. Section 3 deals with the intersection Lawson homology version of the Lawson Suspension Theorem, and in Section 4 we discuss the intersection Lawson homology analogue of the Friedlander-Mazur s-operation.

\section{ACKNOWLEDGMENTS}

I am very grateful to Blaine Lawson for suggesting the problem of finding an intersection version of L-homology and for many stimulating conversations, which were extremely useful in the development of this work. I wish to thank Eric Friedlander for his very helpful comments, suggestions, and encouragement during preparation of this paper. I also thank the referee for his useful remarks and suggestions. I am especially grateful to Paulo Lima-Filho for the persistence with which he encouraged me to finish this paper, and for his help in filling up a gap in the proof of the Intersection Lawson Suspension Theorem. I am indebted to The Mathematical Sciences Research Institute in Berkeley, where most of the work on intersection Lawson homology has been done, for its hospitality and support.

\section{Basic constructions}

In this section we define intersection Lawson homology and discuss some elementary properties of these groups. In this paper by a projective variety we mean a reduced scheme over $\mathbb{C}$ admitting a closed embedding in some complex projective space $\mathbb{P}_{n}$.

1.1. Perversity $\bar{p}$ Lawson filtrations. A filtered space $(X, X)$ is a space $X$ with a filtration

$$
x: \quad X=X^{0} \supset X^{1} \supset \cdots \supset X^{n} \supset X^{n+1}=\emptyset
$$

of $X$ by closed (if $X$ is of finite dimension) subsets of $X$. We refer to the element $X^{k}$ of the filtration $X$ as the formal codimension $k$ skeleton of $X$. The differences $X^{k}-X^{k+1}$ are called strata of $X$. The codimension zero stratum $X-X^{1}$ is called the regular stratum of $\mathcal{X}$. The most important examples of filtrations are different types of stratifications (decompositions of a space into disjoint unions of manifolds). Every complex quasi-projective variety admits a Whitney stratification [Wh1, Wh2, Lo]. Moreover, for every complex quasi-projective variety there is a unique stratification, the canonical Whitney stratification, which is the coarsest in the class of all Whitney stratifications on the given variety [Tr]. Recall, that a stratification $X$ coarsens another stratification $X^{\prime}$ of $X$ if every stratum of $X$ is 
the union of connected components of strata of $X^{\prime}$. For example, if $X$ is a quasiprojective variety of dimension $n$ with isolated singularities $\Sigma=\left\{x_{0}, \ldots, x_{n}\right\}$, then the canonical Whitney stratification takes the form (5) with $X^{i}=\Sigma$ for $0<i \leq n$. A generic Whitney stratification of the variety $X$ may have some extra skeleta between $X$ and $\Sigma$.

Let $X$ be a stratification of a variety. The $l$-skeleton $Z_{k}(X)^{l}$ of the Lawson filtration

$$
Z_{0}(X): \quad Z_{0}(X)=Z_{0}(X)^{0} \supset Z_{0}(X)^{1} \supset Z_{0}(X)^{2} \supset \cdots \supset Z_{0}(X)^{n} \supset \emptyset,
$$

of $Z_{k}(X)$, associated with $X$, consists of the 0 -cycles $\sum n_{i} x_{i}$ of $X$ so that

$$
\operatorname{dim}\left(\left|\sum n_{i} x_{i}\right| \cap X^{l}\right) \geq 0 .
$$

The way one can extend the definition of Lawson filtration from spaces of zero cycles to higher dimensional cycles becomes clear if one rewrites the definition of the Lawson filtration of $Z_{0}(X)$ in the following form. $\sum n_{i} x_{i} \in Z_{k}(X)^{l}$ if there is $j \geq l$ so that

$$
\operatorname{dim}\left(\left|\sum n_{i} x_{i}\right| \cap X^{j}\right) \geq \operatorname{dim}\left(\sum n_{i} x_{i}\right)-j+\bar{t}_{c}(j)+1
$$

where $\bar{t}_{c}$ is the complex top perversity so that $\bar{t}_{c}(0)=\bar{t}_{c}(1)=0$ and $\bar{t}_{c}(i)=i-1$ for $i \geq 1$. Replacing, in the above definition of Lawson filtration, the zero cycle $\sum n_{i} x_{i}$ by a cycle $c$ of dimension $k \geq 0$ and the complex top perversity $\bar{t}_{c}$ by a perversity $\bar{p}$, we get the following definition of perversity $\bar{p}$ Lawson filtration:

$$
Z_{k}(X)^{\bar{p}}: \quad Z_{k}(X)=Z_{k}(X)^{\bar{p}, 0} \supset Z_{k}(X)^{\bar{p}, 1} \supset Z_{k}(X)^{\bar{p}, 2} \supset \cdots \supset Z_{k}(X)^{\bar{p}, n} \supset \emptyset
$$

of $Z_{k}(X)$ induced by the stratification $X$. $c \in Z_{k}(X)^{\bar{p}, l}$ if there is $j \geq l$ so that

$$
\operatorname{dim}\left(|c| \cap X^{j}\right) \geq k-j+\bar{p}(j)+1 .
$$

In other words, $c \in Z_{k}(X)^{\bar{p}, l}$ if $c$ violates the perversity $\bar{p}$ condition

$$
\operatorname{dim}\left(|c| \cap X^{j}\right) \leq k-j+\bar{p}(j),
$$

on a skeleton $X^{j}$ of $X$ of codimension $j \geq l$. The perversity $\bar{p}$ Lawson filtration $Z_{k}(X)^{\bar{p}}$ of $Z_{k}(X)$ induces a perversity $\bar{p}$ Lawson filtration $\mathcal{C}_{k}(X)^{\bar{p}}$ of the monoid

$$
\mathfrak{C}_{k}(X)=\coprod_{r+s \geq 0} \mathfrak{e}_{k, r}(X) \times \mathfrak{C}_{k, s}(X),
$$

of effective cycles of dimension $k$ on $X$, where $\mathcal{C}_{k, r}(X)$ is the Chow variety of degree $r k$-cycles of $X \subset \mathbb{P}_{n}$.

In the sequel, we will be dealing only with the canonical perversity $\bar{p}$ Lawson filtration $Z_{k}\left(X_{c a n}\right)^{\bar{p}}$, which, by the abuse of notation, will be denoted by $Z_{k}(X)^{\bar{p}}$.

If $X$ is a complex projective surface, then the space of 1-cycles $Z_{1}(X)$ of $X$ has only two Lawson filtrations corresponding to the zero and the complex top perversities.

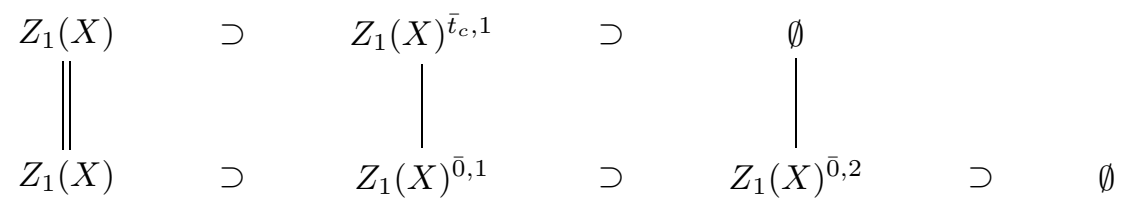

where $Z_{1}(X)^{\bar{t}_{c}, 1}$ consists of 1-cycles of $X$ with at least one component contained in the canonical codimension one skeleton $X_{c a n}^{1}$ of $X, Z_{1}(X)^{\overline{0}, 2}$ consists of 1-cycles 
of $X$ with at least one component intersecting the codimension two skeleton $X_{\text {can }}^{2}$, and

$$
Z_{1}(X)^{\overline{0}, 1}=Z_{1}(X)^{\bar{t}_{c}, 1} \cup Z_{1}(X)^{\overline{0}, 2} .
$$

1.2. Intersection homotopy groups. Before we define intersection Lawson homology we recall the definition and basic properties of intersection homotopy groups. For more details on intersection homotopy groups we refer the reader to [Gr].

Let $P$ be a polyhedron of dimension $k$ and let $X$ be a space with a finite filtration

$$
X: \quad X=X^{0} \supset X^{1} \supset \cdots \supset X^{n} \supset \emptyset .
$$

A continuous map $f: P \rightarrow X$ is $\bar{p}$ allowable with respect to $X$ if for every skeleton $X^{s}$ of $X$ the subset $f^{-1}\left(X^{s}\right)$ is contained in a subpolyhedron of $P$ of dimension less than or equal to $k-s+\bar{p}(s)$. In the sequel, we will sometimes abuse the notation and write $\operatorname{dim} f^{-1}\left(X^{s}\right) \leq m$ instead of saying that $f^{-1}\left(X^{s}\right)$ is contained in a subpolyhedron of $P$ of dimension less than or equal to $m$.

Let $M$ be a pl manifold of dimension $k$. A continuous map $f: M \rightarrow X$ is of perversity $\bar{p}$ with respect to a filtration $\mathcal{X}$ of $X$, or perversity $\bar{p}$ (rel $\mathcal{X}$ ), if $f$ and its restriction $\left.f\right|_{\partial M}$ to the boundary $\partial M$ of $M$ are both $\bar{p}$ allowable maps with respect to $X$. Two maps $f_{0}, f_{1}: M \rightarrow X$ are $\bar{p}$-homotopic if there is a homotopy $F: M \times I \rightarrow X$ between $f_{0}$ and $f_{1}$, which is a perversity $\bar{p}$ map.

Let $(X, X)$ be a filtered space with a base point $x$ contained in the top dimensional stratum $X^{0}-X^{1}$ of $X$. The $r$-th perversity $\bar{q}$ intersection homotopy group $I_{\bar{q}} \pi_{r}(X, X ; x)$ of $(X, X)$ consists of $\bar{q}$-homotopy classes of perversity $\bar{q}$ maps $\left(\mathbb{S}_{r}, s\right) \rightarrow(X, x)$ where $s$ is the south pole of the unit sphere $\mathbb{S}_{r}$ of dimension $r$.

The addition of elements of $I_{\bar{q}} \pi_{r}(X, X)$ is defined as in the ordinary homotopy groups. It is well defined (it preserves the perversity $\bar{q}$ condition), because the base point $x$ of $X$ is in the top dimensional stratum of $X$.

In the sequel we will use another definition of intersection homotopy groups given in terms of simplicial set theory.

Let $\Delta^{r}$ be the standard $k$-dimensional simplex. A perversity $\bar{q}$ singular simplex of a filtered space $(X, X)$ is a continuous map $\sigma: \Delta^{r} \rightarrow X$ so that for every face $\Delta$ of $\Delta^{r}$ the inverse image $\left(\left.\sigma\right|_{\Delta}\right)^{-1}\left(X^{l}\right)$ is contained in a subpolyhedron of $\Delta$ of codimension greater than or equal to $l-\bar{q}(l)$ in $\Delta$. Let $I_{\bar{q}} S_{r}(X, X)$ be the set of all perversity $\bar{q}$ singular $r$-simplexes of the filtered space $(X, X)$ and let

$$
I_{\bar{q}} S(X, X)=\coprod_{r \geq 0} I_{\bar{q}} S_{r}(X, X)
$$

be the simplicial complex of perversity $\bar{q}$ singular simplexes of $(X, X)$. Directly from the definition of simplicial homotopy groups it follows that for every perversity $\bar{q}$ and every $r>0$ there is an isomorphism

$$
I_{\bar{q}} \pi_{r}(X, X) \cong \pi_{r}\left(I_{\bar{q}} S(X, X)\right)
$$

which is an intersection analogue of the classical isomorphism

$$
\pi_{r}(X) \cong \pi_{r}(S(X)) \text {. }
$$

A map $f:(X, X) \rightarrow(Y, Y)$ of filtered spaces is called filtered (resp. cofiltered) if for every $l \geq 0$ we have $f^{-1}\left(Y^{l}\right) \supset X^{l}$ (resp. $\left.f^{-1}\left(Y^{l}\right) \subset X^{l}\right)$. If $f: X \rightarrow Y$ is a cofiltered map, then $f$ induces a simplicial map

$$
f_{\sharp}: I_{\bar{q}} S(X, X) \rightarrow I_{\bar{q}} S(Y, y)
$$


and hence a homomorphism

$$
f_{*}: I_{\bar{q}} \pi_{*}(X, X) \rightarrow I_{\bar{q}} \pi_{*}(Y, y) .
$$

A pair $((X, X),(A, \mathcal{A}))$ is called a filtered pair if an embedding $i: A \hookrightarrow X$ is a cofiltered map. For example, if $x \in X$, then $((X, X), x)$ is a filtered pair if and only if $x$ is an element of the regular stratum of $X$. A triple $((X, X),(A, \mathcal{A}),(B, \mathcal{B}))$ is called a filtered triple if both pairs $((X, X),(A, \mathcal{A})),((A, \mathcal{A}),(B, \mathcal{B}))$ are filtered pairs.The $r$-th perversity $\bar{q}$ intersection homotopy group $I_{\bar{q}} \pi_{r}((X, X),(A, \mathcal{A}) ; x)$ of a filtered triple $((X, X),(A, \mathcal{A}), x)$ is the $r$-th simplicial homotopy group of the simplicial triple $\left(I_{\bar{q}} S(X, X), I_{\bar{q}} S(A, \mathcal{A}), I_{\bar{q}} S(x)\right)$.

For every filtered triple $((X, X),(A, \mathcal{A}), x)$ there is a long exact sequence of intersection homotopy groups

$$
\cdots \rightarrow I_{\bar{q}} \pi_{r}(A, \mathcal{A}) \rightarrow I_{\bar{q}} \pi_{r}(X, X) \rightarrow I_{\bar{q}} \pi_{r}((X, X),(A, \mathcal{A})) \rightarrow I_{\bar{q}} \pi_{r-1}(A, \mathcal{A}) \rightarrow \cdots
$$

A map $\pi:(E, \mathcal{E}) \rightarrow(B, \mathcal{B})$ of filtered spaces will be called a filtered fibration if for every perversity $\bar{q}$ it induces a simplicial fibration $\pi_{\bar{q}}: I_{\bar{q}} S(E, \mathcal{E}) \rightarrow I_{\bar{q}} S(B, \mathcal{B})$. If $\pi:(E, \mathcal{E}) \rightarrow(B, \mathcal{B})$ is a filtered fibration so that for some $b$ in a regular stratum of $B$ the fiber $F=\pi^{-1}(b)$ is a filtered subspace of $E$, then there exists a long exact sequence of intersection homotopy groups

$$
\cdots \rightarrow I_{\bar{q}} \pi_{r}(F, \mathcal{F} ; e) \rightarrow I_{\bar{q}} \pi_{r}(E, \mathcal{E} ; e) \rightarrow I_{\bar{q}} \pi_{r}(B, \mathcal{B} ; b) \rightarrow I_{\bar{q}} \pi_{r-1}(F, \mathcal{F} ; e) \rightarrow \cdots
$$

1.3. Intersection Lawson homology. Let $X$ be a complex projective variety. The group $Z_{k}(X)$ of algebraic $k$-cycles of $X$ has a natural topology induced by the quotient map

$$
\mathcal{C}_{r}(X) \times \mathcal{C}_{r}(X) \longrightarrow Z_{r}(X), \quad\left(c, c^{\prime}\right) \mapsto c-c^{\prime},
$$

where

$$
\mathcal{C}_{r}(X)=\coprod_{d \geq 0} \mathfrak{e}_{r, d}(X)
$$

is the Chow monoid with $\mathcal{C}_{r, d}(X)$ being the Chow variety of effective $r$-cycles of degree $d$ in $X$ [L1]. For a quasi-projective variety $U \subset \mathbb{P}^{n}$ with closure $\bar{U}$ we set

$$
Z_{k}(U) \stackrel{\text { def }}{=} Z_{k}(\bar{U}) / Z_{k}(\bar{U}-U) .
$$

It turns out that the homeomorphism type of the space $Z_{k}(U)$ does not depend on the closure $\bar{U}$ (for more details on Lawson homology of quasi-projective varieties see $[\mathrm{LF} 1])$.

The intersection Lawson homology groups of a complex projective variety $X$ are the intersection homotopy groups

$$
I_{\bar{q}} \pi_{r}\left(Z_{k}(X), Z_{k}(X)^{\bar{p}}\right)=\pi_{r}\left(I_{\bar{q}} S\left(Z_{k}(X), Z_{k}(X)^{\bar{p}}\right)\right) .
$$

If $V \subset X$ is a pair of projective varieties, then we define the relative intersection Lawson homology groups of the pair $(X, V)$ by

$$
I_{\bar{q}} \pi_{r}\left(Z_{k}(X, V), Z_{k}(X, V)^{\bar{p}}\right),
$$

where $Z_{k}(X, V)^{\bar{p}}$ is the image of the canonical perversity $\bar{p}$ filtration $Z_{k}(X)^{\bar{p}}$ of $Z_{k}(X)$ by the projection

$$
Z_{k}(X) \longrightarrow Z_{k}(X, V) \stackrel{\text { def }}{=} Z_{k}(X) / Z_{k}(V) .
$$


By the abuse of notation we will denote by $I_{\bar{q}} \pi_{r}\left(Z_{k}(X)^{\bar{p}}\right)$ the intersection Lawson homology of $X$ and by $I_{\bar{q}} \pi_{r}\left(Z_{k}(X, V)^{\bar{p}}\right)$ the relative intersection Lawson homology of the pair $(X, V)$.

Examples. 1. Let $\bar{q}$ be a perversity so that $\bar{q}(0)=\bar{q}(1)=0$. Then for every filtered space $\left(X,\left\{X^{l}\right\}_{l \geq 0}\right)$

$$
I_{\bar{q}} \pi_{0}\left(X,\left\{X^{l}\right\}_{l \geq 0}\right)=\pi_{0}\left(X-X^{1}\right) .
$$

In particular,

$$
I_{\bar{q}} \pi_{0}\left(Z_{k}(X)^{\bar{p}}\right)=\pi_{0}\left(Z_{k}(X)-Z_{k}(X)^{\bar{p}, 1}\right)=\pi_{0}\left(I_{\bar{p}} Z_{k}(X)\right) .
$$

The group $\pi_{0}\left(I_{\bar{p}} Z_{k}(X)\right)$ can be identified with the quotient of $Z_{k}(X)$ by a perversity $\bar{p}$ algebraic equivalence relation, where two $k$-cycles $c_{0}, c_{1}$ of $X$ are perversity $\bar{p}$ algebraic equivalent if there are a non-singular variety $T$ of dimension $m$ and a finite family of subvarieties $W_{i}$ of $X \times T$ of dimension $m+k$ with flat projections $W_{i} \rightarrow T$ so that all fibers of the projections are perversity $\bar{p}$ cycles and there are $t_{0}, t_{1} \in T$ so that $c_{j}$ is the fiber of one of the projections over $t_{j}$ for $j=0,1$. The proof of the isomorphism between $\pi_{0}\left(I_{\bar{p}} Z_{k}(X)\right)$ and the group of perversity $\bar{p}$ algebraic equivalence classes of perversity $\bar{p} k$-cycles of $X$ is an easy modification of the proof from $[\mathrm{Fr}]$.

2. Let $X$ be a complex projective variety of dimension $n$ with isolated singularities $\Sigma=\left\{x_{0}, \ldots, x_{n}\right\}$. Then for every $k \geq 0$ there is only one non-trivial Lawson filtration

$$
Z_{k}(X)=Z_{k}(X)^{0} \supset Z_{k}(X)^{1} \supset Z_{k}(X)^{2} \supset \cdots \supset Z_{k}(X)^{n} \supset \emptyset
$$

of $Z_{k}(X)$ so that

$$
Z_{k}(X)^{i}=\left\{c \in Z_{k}(X) \mid \operatorname{dim}(|c| \cap \Sigma) \neq \emptyset\right\}
$$

for $0<i \leq n$. The intersection homotopy groups of $Z_{k}(X)$ with respect to this filtration will be denoted simply by $I_{\bar{q}} \pi_{r}\left(Z_{k}(X)\right)$. The intersection fundamental groups of $Z_{k}(X)$ are given by the following formula:

$$
I_{\bar{q}} \pi_{1}\left(Z_{k}(X)\right) \cong \begin{cases}\pi_{1}\left(\left.Z_{k}(X)\right|_{X-\Sigma}\right) & \text { for } 2-n+\bar{q}(n)<0 \\ \operatorname{im}\left(\pi_{1}\left(Z_{k}(X)\right) \longrightarrow \pi_{1}\left(\left.Z_{k}(X)\right|_{X-\Sigma}\right)\right) & \text { for } 2-n+\bar{q}(n)=0 \\ \pi_{1}\left(Z_{k}(X)\right) & \text { for } 2-n+\bar{q}(n)>0\end{cases}
$$

where $\left.Z_{k}(X)\right|_{X-\Sigma}$ is the subgroup of $Z_{k}(X)$ consisting of cycles with support in $X-\Sigma$. For higher dimensional intersection homotopy groups of $Z_{k}(X)$ it is easy to see that

$$
I_{\bar{q}} \pi_{r}\left(Z_{k}(X)\right) \cong \pi_{r}\left(\left.Z_{k}(X)\right|_{X-\Sigma}\right) \quad \text { for } r+1-n+\bar{q}(n)<0,
$$

but for other values of $r+1-n+\bar{q}(n)$ the groups $I_{\bar{q}} \pi_{r}\left(Z_{k}(X)\right)$ are difficult to compute.

Recall that in all our considerations we deal with the canonical (the coarsest) Whitney stratifications of complex varieties. Thus, when talking about filtered or cofiltered maps between complex varieties we always mean filtered or cofiltered maps with respect to these canonical stratifications. Under the above assumption, the class of complex varieties together with filtered (resp. cofiltered) maps form a category. Now we are going to show that intersection Lawson homology is a covariant (resp. contravariant) functor on this category. In what follows all spaces are complex projective varieties. 
Proposition 1.1. Any proper cofiltered map $f: X \rightarrow Y$ of projective varieties induces a homomorphism

$$
f_{*}: I_{\bar{q}} \pi_{r}\left(Z_{k}(X)^{\bar{p}}\right) \longrightarrow I_{\bar{q}} \pi_{r}\left(Z_{k}(Y)^{\bar{p}}\right) .
$$

Moreover, if $f: X \rightarrow Y$ and $g: Y \rightarrow Z$ are both proper and cofiltered maps of projective varieties, then $(g \circ f)_{*}=g_{*} \circ f_{*}$.

Proof. Since $f$ is proper it induces a continuous map [LF2]

$$
f_{\sharp}: Z_{r}(X) \longrightarrow Z_{r}(Y) .
$$

We will show that for every perversity $\bar{p}$ the map $f_{\sharp}$ is cofiltered with respect to perversity $\bar{p}$ filtrations on $Z_{r}(X)$ and $Z_{r}(Y)$ and hence induces a homomorphism of intersection homotopy groups. We want to show that for an arbitrary $\bar{p}$ and every $l \geq 0$

$$
f_{\sharp}^{-1}\left(Z_{r}(Y)^{\bar{p}, l}\right)=f_{\sharp}^{-1}\left(f_{\sharp}\left(Z_{r}(X)\right) \cap Z_{r}(Y)^{\bar{p}, l}\right) \subset Z_{r}(X)^{\bar{p}, l} .
$$

In other words, we want to prove that if for some $r$-cycle $c$ of $X$ and some $i \geq l$

$$
\operatorname{dim}\left(\left|f_{\sharp}(c)\right| \cap Y^{i}\right) \geq(r-i)+\bar{p}(i)+1,
$$

then

$$
\operatorname{dim}\left(|c| \cap X^{i}\right) \geq(r-i)+\bar{p}(i)+1
$$

where dim denotes complex dimension of the corresponding complex algebraic sets.

The above implication results from the following sequence of equalities and inequalities

$$
\begin{aligned}
\operatorname{dim}\left(|c| \cap X^{i}\right) & \geq \operatorname{dim}\left(\left|f_{\sharp}(c)\right| \cap f\left(X^{i}\right)\right) \geq \operatorname{dim}\left(\left|f_{\sharp}(c)\right| \cap f(X) \cap Y^{i}\right) \\
& =\operatorname{dim}\left(\left|f_{\sharp}(c)\right| \cap Y^{i}\right)
\end{aligned}
$$

where the second inequality follows from the cofiltration property of $f$.

Proposition 1.2. Let $X$ and $Y$ be projective varieties. Any filtered flat map $f$ : $X \rightarrow Y$ of relative dimension $s$ induces a homomorphism

$$
f^{*}: I_{\bar{q}} \pi_{r}\left(Z_{k}(Y)^{\bar{p}}\right) \longrightarrow I_{\bar{q}} \pi_{r}\left(Z_{k+s}(X)^{\bar{p}}\right) .
$$

Moreover, if $f: X \rightarrow Y$ and $g: Y \rightarrow Z$ are both flat and filtered, then $(g \circ f)^{*}=$ $f^{*} \circ g^{*}$.

Proof. If $f: X \rightarrow Y$ of relative dimension $s$, then it induces a continuous map [LF2]

$$
f^{\sharp}: Z_{r}(Y) \longrightarrow Z_{r+s}(X) .
$$

We will see that for every perversity $\bar{p}$ this is a cofiltered map with respect to perversity $\bar{p}$ filtrations on $Z_{r+s}(X)$ and $Z_{r}(Y)$. Actually, the inclusion

$$
\left(f^{\sharp}\right)^{-1}\left(Z_{r+s}(X)^{\bar{p}, l}\right) \subset Z_{r}(Y)^{\bar{p}, l}
$$

is a consequence of the following sequence of equalities and inequalities:

$$
\begin{aligned}
\operatorname{dim}\left(|c| \cap Y^{i}\right)+s & =\operatorname{dim}\left(f^{\sharp}\left(|c| \cap Y^{i}\right)\right)=\operatorname{dim}\left(\left|f^{\sharp}(c)\right| \cap f^{-1}\left(Y^{i}\right)\right) \\
& \geq \operatorname{dim}\left(\left|f^{\sharp}(c)\right| \cap X^{i}\right) .
\end{aligned}
$$




\section{Localization SEQUence}

Recall that a pair $(X, V)$ of projective varieties is a stratified pair if $V^{l}=V \cap X^{l}$ for every $l \geq 0$. For example, if $V$ is a generic hyperplane section of $X$, then $(X, V)$ is a stratified pair. If $(X, V)$ is a stratified pair, then there is a long exact sequence of intersection homology groups

$$
\cdots \rightarrow I_{\bar{q}} H_{r}(V) \rightarrow I_{\bar{q}} H_{r}(X) \rightarrow I_{\bar{q}} H_{r}(X, V) \rightarrow I_{\bar{q}} H_{r-1}(V) \rightarrow \cdots .
$$

The aim of this section is to prove the following refinement of the above result.

Theorem 2.1. If $X$ is a projective variety and $(X, V)$ is a stratified pair, then there exists a long exact sequence

$\cdots \rightarrow I_{\bar{q}} \pi_{r}\left(Z_{k}(V)^{\bar{p}}\right) \rightarrow I_{\bar{q}} \pi_{r}\left(Z_{k}(X)^{\bar{p}}\right) \rightarrow I_{\bar{q}} \pi_{r}\left(Z_{k}(X, V)^{\bar{p}}\right) \rightarrow I_{\bar{q}} \pi_{r-1}\left(Z_{k}(V)^{\bar{p}}\right) \rightarrow \cdots$

where $Z_{k}(X, V)^{\bar{p}}$ is the image of the canonical perversity $\bar{p}$ filtration $Z_{k}(X)^{\bar{p}}$ of $Z_{k}(X)$ by the projection

$$
Z_{k}(X) \longrightarrow Z_{k}(X, V) \stackrel{\text { def }}{=} Z_{k}(X) / Z_{k}(V) .
$$

Remarks. 1. The above long exact sequence specializes to the intersection homology long exact sequence (6) for $\bar{q}=\bar{t}_{c}$ and $k=0$.

2. If $X$ is a quasi-projective variety and $(X, V)$ is a stratified pair so that $X$ has a projective closure $\bar{X}$ so that $(\bar{X}, \bar{X}-X)$ and $(\bar{X}, \bar{V}-X)$ are stratified pairs, then the standard diagram chasing argument involving long exact sequences of $(\bar{X}, \bar{X}-X),(\bar{X}, \bar{V}-X)$, and $(\bar{X}-X, \bar{V}-X)$ gives an intersection Lawson homology localization sequence for the pair $(X, V)$.

Proof. Recall that a map $\pi: E \rightarrow B$ of filtered spaces is a filtered fibration if for every perversity $\bar{q}$ it induces a simplicial fibration

$$
\pi_{\bar{q}}: I_{\bar{q}} S(E) \longrightarrow I_{\bar{q}} S(B) \text {. }
$$

Filtered fibrations are intersection homotopy-theoretic analogues of fibrations in the sense that they induce long exact sequences of intersection homotopy groups.

Theorem 2.2 ([Gr]). If $\pi: E \rightarrow B$ is a filtered fibration so that for some $b$ in a regular stratum of $B$ the fiber $F=\pi^{-1}(b)$ is a filtered subspace of $E$, then there exists a long exact sequence of intersection homotopy groups

$$
\cdots \rightarrow I_{\bar{q}} \pi_{r}(F, e) \rightarrow I_{\bar{q}} \pi_{r}(E, e) \rightarrow I_{\bar{q}} \pi_{r}(B, b) \rightarrow I_{\bar{q}} \pi_{r-1}(F, e) \rightarrow \cdots .
$$

In order to prove Theorem 2.1 it is enough to show that every stratified pair $(X, V)$ and every perversity $\bar{q}$ the projection

$$
\pi: Z_{k}(X) \longrightarrow Z_{k}(X, V)
$$

is a filtered fibration with respect to perversity $\bar{q}$ filtrations of $Z_{k}(X)$ and $Z_{k}(X, V)$ and that the fiber $Z_{k}(V)$ of $\pi$ is a filtered subspace of $Z_{k}(X)$. The last statement is a trivial consequence of the fact that $(X, V)$ is a stratified pair. In order to prove that $\pi: Z_{k}(X) \longrightarrow Z_{k}(X, V)$ is a filtered fibration we will use the following result.

Theorem $2.3([\mathrm{Gr}])$. Let $\pi: E \rightarrow B$ be a locally trivial fibration with fiber $F$ and let $\left\{E^{i}\right\},\left\{B^{i}\right\}$, and $\{F\}$ be filtrations of $E, B$, and $F$ respectively. If there is a covering $\mathcal{U}$ of $B$ so that for every $U \in \mathcal{U}$ there is a trivialization $\varphi: \pi^{-1}(U) \rightarrow U \times F$ so that for every $l \geq 0$ and $U^{l}=U \cap B^{l}$ 


$$
\varphi\left(\pi^{-1}(U) \cap E^{l}\right)=U^{l} \times F \cup U \times F^{l},
$$

then $\pi$ is a filtered fibration.

Actually, in the case when $E$ is a topological group and $B$ is its quotient group it is enough to show that there is a single neighborhood $U$ of 0 in $B$ for which the condition (7) is satisfied. The required covering of $B$ is obtained by the action of $E$ on $U$ and the appropriate change of the trivialization map for $\pi^{-1}(U)$. Thus we have to prove the following result.

Proposition 2.4. There is a neighborhood $U$ of 0 in $Z_{k}(X, V)$ and a trivialization

$$
\varphi: \pi^{-1}(U) \longrightarrow U \times Z_{k}(V)
$$

so that for every $l \geq 0$ and $U^{\bar{q}, l}=U \cap Z_{k}(X, V)^{\bar{q}, l}$

$$
\varphi\left(\pi^{-1}(U) \cap Z_{k}(X)^{\bar{q}, l}\right)=U^{\bar{q}, l} \times Z_{k}(V) \cup U \times Z_{k}(V)^{\bar{q}, l} .
$$

In the course of the proof of Proposition 2.4 we will use the following notation.

$$
\begin{gathered}
\mathcal{C}=\mathcal{C}_{k}(X), \quad \mathcal{Z}=Z_{k}(X), \quad \mathcal{C}^{\prime}=\mathcal{C}_{k}(V), \quad Z^{\prime}=Z_{k}(V), \quad \mathcal{Q}=\mathcal{Z} / \mathcal{Z}^{\prime}, \\
p: \mathcal{C} \times \mathcal{C} \longrightarrow \mathcal{Z}, \pi: \mathcal{Q} \text { are the quotient maps, } \\
(\mathcal{C} \times \mathcal{C})_{d}=\left(\mathcal{C}_{k}(X) \times \mathcal{C}_{k}(X)\right)_{d}=\bigcup_{s+l \leq d} \mathcal{C}_{r, s}(X) \times \mathcal{C}_{r, l}(X), \\
Z_{d}=p\left((\mathcal{C} \times \mathcal{C})_{d}\right), \quad Q_{d}=\pi\left(Z_{d}\right), \\
z^{\bar{q}, l}=Z_{k}(X)^{\bar{q}, l}, \quad z_{d}^{\bar{q}, l}=Z_{d} \cap Z^{\bar{q}, l}, \\
(\mathcal{C} \times \mathcal{C})^{\bar{q}, l}=p^{-1}\left(Z^{\bar{q}, l}\right), \quad(\mathcal{C} \times \mathcal{C})_{d}^{\bar{q}, l}=(\mathcal{C} \times \mathcal{C})_{d} \cap(\mathcal{C} \times \mathcal{C})^{\bar{q}, l}, \\
\mathbb{Q}^{\bar{q}, l}=\pi\left(\mathcal{Z}^{\bar{q}, l}\right) .
\end{gathered}
$$

Proof of Proposition 2.4. We want to construct a neighborhood $U$ of 0 in $Q$ and a section $s: U \rightarrow Z$ of $\pi: Z \rightarrow Q$ so that the associated projection onto the fiber

$$
\begin{aligned}
& \sigma: \pi^{-1}(U) \longrightarrow Z^{\prime}, \\
& \sigma(x)=s(\pi(x))-x
\end{aligned}
$$

induces a trivialization

$$
(\pi, \sigma): \pi^{-1}(U) \longrightarrow U \times z^{\prime}
$$

so that

$$
(\pi, \sigma)\left(\pi^{-1}(U) \cap Z^{\bar{q}, l}\right)=U^{\bar{q}, l} \times Z^{\prime} \cup U \times\left(Z^{\prime}\right)^{\bar{q}, l}
$$

where $U^{\bar{q}, l}=U \cap Q^{\bar{q}, l}$.

zWe will construct a family $\left\{U_{d}\right\}$ of open neighborhoods of 0 in $Q_{d}$ and sections

$$
s_{d}: U_{d} \longrightarrow z
$$

so that

$$
\left.U_{d}\right|_{Q_{d-1}}=U_{d-1} \text { and }\left.s_{d}\right|_{U_{d-1}}=s_{d-1}
$$

and

$$
\left(\pi, \sigma_{d}\right)\left(\pi^{-1}\left(U_{d}\right) \cap Z_{d}^{\bar{q}, l}\right)=U_{d}^{\bar{q}, l} \times Z^{\prime} \cup U_{d} \times\left(Z_{d}^{\prime}\right)^{\bar{q}, l}
$$


where $U_{d}^{\bar{q}, l}=U_{d} \cap Q^{\bar{q}, l}$ and $\sigma_{d}$ is the following projection onto the fiber:

$$
\begin{gathered}
\sigma_{d}: Z_{d} \cap \pi^{-1}\left(U_{d}\right) \longrightarrow \mathcal{Z}^{\prime}, \\
\sigma_{d}(x)=s_{d}(\pi(x))-x .
\end{gathered}
$$

Then we set $U=\bigcup U_{d}$ and $s: U \rightarrow \mathcal{Z}$ so that $\left.s\right|_{U_{d}}=s_{d}$. The properties (10), (11) of $s_{d} \mathrm{~S}$ imply that $s$ is well defined and satisfies (9).

Since $Q_{0}=0$ we set $U_{0}=0$ and $s_{0}(0)=0$. Suppose, we have already constructed an open neighborhood $U_{d-1}$ of 0 in $Q_{d-1}$ and a section of $\pi$

$$
s_{d-1}: U_{d-1} \longrightarrow z
$$

that satisfies the conditions (10) and (11). Let us consider an extension of $s_{d-1}$ to a map

$$
\sigma_{d-1}: z_{d} \cap \pi^{-1}\left(U_{d-1}\right) \longrightarrow Z^{\prime}
$$

given by the same formula as in (12).

We will extend $\sigma_{d-1}$ to a map $\sigma_{d}$ on a neighborhood $W_{d}$ of 0 in $z_{d}$, set $U_{d}=$ $\pi\left(W_{d}\right)$, and show that $s_{d}: U_{d} \longrightarrow \mathcal{Z}$ given by $s_{d}(\pi(x))=\sigma_{d}(x)+x$ is a well defined required section. We will choose $W_{d}$ so that there is a retraction

$$
r_{d}: W_{d} \longrightarrow z_{d} \cap \pi^{-1}\left(U_{d-1}\right)
$$

and then define $\sigma_{d}=\sigma_{d-1} \circ r_{d}$.

The next few paragraphs are devoted to a construction of a retraction $r_{d}$ so that the resulting trivialization will have the required properties.

First, note that $(\mathcal{C} \times \mathcal{C})_{d} \cap(\pi \circ p)^{-1}\left(Q_{d-1}\right)$ is a Zariski closed subset of $(\mathcal{C} \times \mathcal{C})_{d}$. Actually, it consists of those $\left(c, c^{\prime}\right) \in(\mathcal{C} \times \mathcal{C})_{d}$ so that $c$ or $c^{\prime}$ has a component contained in $V$.

Every complex projective variety $X$ has a triangulation. Moreover, for any family of semialgebraic subsets $V_{1}, V_{2}, \ldots, V_{n}$ of $X$ there is a triangulation of $X$ compatible with $V_{1}, V_{2}, \ldots, V_{n}$ [Hi]. Applying the above theorem to $(\mathcal{C} \times \mathcal{C})_{d},(\mathcal{C} \times \mathcal{C})_{d} \cap$ $(\pi \circ p)^{-1}\left(Q_{d-1}\right)$, and the closures of $(\mathcal{C} \times \mathcal{C})_{d}^{\bar{q}, l}$ for $l \geq 0$ we get a triangulation of $(\mathcal{C} \times \mathcal{C})_{d}$ compatible with these subsets. Since $(\mathcal{C} \times \mathcal{C})_{d} \cap(\pi \circ p)^{-1}\left(Q_{d-1}\right)$ is a subpolyhedron of $(\mathcal{C} \times \mathcal{C})_{d}$ it is a NDR of $(\mathfrak{C} \times \mathfrak{C})_{d}$.

Lemma 2.5. Let $X$ be a polyhedron and let $A, B_{1} \subset B_{2} \subset \cdots \subset B_{n}$ be its closed subpolyhedra. Then there are an open neighborhood $U$ of $A$ in $X$ and a retraction $r: U \rightarrow A$ so that for every $i \in\{0,1, \ldots, n\}$

$$
r\left(U \cap\left(B_{i}-B_{i-1}\right)\right) \subset A \cap\left(B_{i}-B_{i-1}\right) .
$$

Proof. Fix a triangulation of $X$ compatible with $A$ and all $B_{i}$ s. Let $U$ be a derived neighborhood of $A$ in $X$. For every simplex $\Delta$ of $\bar{U}$ the required retraction is given by the projection along the intervals of the join $(\partial \bar{U} \cap \Delta) \star(A \cap \Delta)=\Delta$.

From Lemma 2.5 and the fact that

$$
p:\left((\mathcal{C} \times \mathcal{C})_{d},(\mathcal{C} \times \mathcal{C})_{d} \cap(\pi \circ p)^{-1}\left(Q_{d-1}\right)\right) \longrightarrow\left(z_{d}, z_{d} \cap \pi^{-1}\left(Q_{d-1}\right)\right)
$$

is a relative homeomorphism [LF93] it follows that there are a neighborhood $V_{d}$ of $z_{d} \cap \pi^{-1}\left(Q_{d-1}\right)$ in $z_{d}$ and a retraction

$$
R_{d}: V_{d} \longrightarrow z_{d} \cap \pi^{-1}\left(Q_{d-1}\right)
$$


so that

$$
R_{d}\left(V_{d} \cap\left(z_{d}^{\bar{q}, l}-z_{d}^{\bar{q}, l+1}\right)\right) \subset\left(z_{d}^{\bar{q}, l}-z_{d}^{\bar{q}, l+1}\right) \cap \pi^{-1}\left(Q_{d-1}\right) .
$$

Let

$$
W_{d}=\left(R_{d}\right)^{-1}\left(Z_{d} \cap \pi^{-1}\left(U_{d-1}\right)\right)
$$

and let $r_{d}$ be the restriction of $R_{d}$ to $W_{d}$. The set $W_{d}$ is open in $z_{d}$ because $Z_{d} \cap \pi^{-1}\left(U_{d-1}\right)$ is open in $Z_{d} \cap \pi^{-1}\left(Q_{d-1}\right)$ and $R_{d}$ is a continuous map. Since $\pi$ is the quotient map the set $U_{d}=\pi\left(W_{d}\right)$ is open in $Q_{d}$. Note that $U_{d} \cap Q_{d-1}=U_{d-1}$.

The map

$$
\begin{gathered}
\sigma_{d}: W_{d} \longrightarrow z^{\prime}, \\
\sigma_{d}=\sigma_{d-1} \circ r_{d}
\end{gathered}
$$

is an extension of $\sigma_{d-1}$ to $W_{d}$. We will show that the map

$$
\begin{gathered}
s_{d}: U_{d} \longrightarrow Z, \\
s_{d}(\pi(x))=\sigma_{d}(x)+x
\end{gathered}
$$

is a well defined section of $\pi$ that satisfies the conditions (10), (11).

In order to prove that $s_{d}$ is well defined we have to show that if $x, x^{\prime} \in W_{d}$ are so that $\pi(x)=\pi\left(x^{\prime}\right)$, then

$$
s_{d}(\pi(x))=s_{d}\left(\pi\left(x^{\prime}\right)\right) .
$$

There are two cases.

- If $\pi(x)=\pi\left(x^{\prime}\right) \in U_{d-1}$, then $\sigma_{d}(x)+x=s_{d-1}(\pi(x))-x+x=s_{d-1}(\pi(x))$.

Hence $\left.s_{d}\right|_{U_{d}-1}=s_{d-1}$ and $s_{d}(\pi(x))=s_{d}\left(\pi\left(x^{\prime}\right)\right)$.

- If $\pi(x)=\pi\left(x^{\prime}\right) \in U_{d}-U_{d-1}$, then $x=x^{\prime}$ because

$$
\pi:\left(W_{d}, z_{d} \cap \pi^{-1}\left(U_{d-1}\right)\right) \longrightarrow\left(U_{d}, U_{d-1}\right)
$$

is a relative homeomorphism.

$s_{d}$ is a section of $\pi$ because

$$
\pi\left(s_{d}(\pi(x))\right)=\pi\left(\sigma_{d}(x)+x\right)=\pi\left(\sigma_{d}(x)\right)+\pi(x)=\pi(x) .
$$

Now we will prove that $s_{d}$ satisfies the condition (11).

First note that

$$
\pi^{-1}\left(U_{d}\right) \cap Z_{d}^{\bar{q}, l}=W_{d} \cap Z^{\bar{q}, l} .
$$

For every element $c$ of $W_{d} \cap Z^{\bar{q}, l}$ there are two possibilities.

A: $c$ has a component $c^{\prime}$ contained in $V$ so that

$$
\operatorname{dim}\left(\left|c^{\prime}\right| \cap X^{l}\right) \geq r-l+\bar{q}(l)+1 .
$$

B: $c$ has no components in $V$ satisfying the above inequality.

In the second case $\pi(c) \in U_{d}^{\bar{q}, l}$ and hence

$$
\left(\pi(c), \sigma_{d}(c)\right) \in U_{d}^{\bar{q}, l} \times Z^{\prime} .
$$

We will show that in the first case $\sigma_{d}(c) \in\left(\mathcal{Z}^{\prime}\right)^{\bar{q}, l}$.

Suppose that $c$ is an element of $z_{d} \cap \pi^{-1}\left(U_{d-1}\right) \subset W_{d} \cap Z^{\bar{q}, l}$, all components of $c$ are contained in $V$, and one of them satisfies the inequality (13). From the definition of $\sigma_{d}$ it follows that $\sigma_{d}(c)=\sigma_{d-1}(c)$. Since all components of $c$ are in $V$ we have $\sigma_{d-1}(c)=-c$ and therefore $\sigma_{d}(c) \in\left(\mathcal{Z}^{\prime}\right)^{\bar{q}, l}$. 
If $c \in Z_{d} \cap \pi^{-1}\left(U_{d-1}\right)$ has a component contained in $V$ that satisfies the inequality (13), but not all of the components of $c$ are in $V$, then we can delete one of the components of $c$ not contained in $V$ and get a cycle $\hat{c} \in Z_{d-1} \cap \pi^{-1}\left(U_{d-1}\right)=W_{d-1}$. We have $\sigma_{d}(c)=\sigma_{d-1}(c)=\sigma_{d-1}(\hat{c})$ with the last equality following directly from the definition of $\sigma_{d-1}$. The induction assumption implies that $\sigma_{d-1}(\hat{c}) \in\left(\mathcal{Z}^{\prime}\right)^{\bar{q}, l}$.

If $c \in W_{d}-\left(Z_{d} \cap \pi^{-1}\left(U_{d-1}\right)\right)$, then $\sigma_{d}(c)=\sigma_{d-1}\left(r_{d}(c)\right)$. Since $r_{d}$ preserves strata of the perversity $\bar{q}$ filtration on $Z_{d}$ the cycle $r_{d}(c) \in Z_{d} \cap \pi^{-1}\left(U_{d-1}\right)$ still satisfies the condition (A). Hence, arguing as above we prove that $\sigma_{d}(c)$ belongs to $\left(Z^{\prime}\right)^{\bar{q}, l}$. This ends the proof of Theorem 2.1.

\section{Intersection Lawson Suspension Theorem}

Let $X \subset \mathbb{P}_{n}$ be a complex projective variety and let $\not X$ be a complex join of $X$ with a point $x_{0}$ in $\mathbb{P}_{n+1}-\mathbb{P}_{n}$, for some linear embedding $\mathbb{P}_{n} \hookrightarrow \mathbb{P}_{n+1}$. We will call $\not{Z} X$ a complex suspension of $X$. The complex suspension induces a continuous map [L2]

$$
\text { \& }: Z_{k}(X) \longrightarrow Z_{k+1}(\not 4 X) \text {. }
$$

One of the central results concerning homotopy type of spaces of cycles is the following Lawson Suspension Theorem.

Theorem 3.1 ([L2]). The map

$$
\text { Z }: Z_{k}(X) \longrightarrow Z_{k+1}(\not X)
$$

is a homotopy equivalence.

Note that $\not X-x_{0}$ is the hyperplane line bundle $\mathcal{O}_{X}(1)$ over $X$. Since

$$
Z_{k+1}\left(\mathcal{O}_{X}(1)\right) \stackrel{\text { def }}{=} Z_{k+1}(\not K X) / Z_{k+1}\left(x_{0}\right) \cong Z_{k+1}(\not K X)
$$

the Lawson Suspension Theorem can be reformulated as follows.

Theorem 3.2. The hyperplane line bundle projection map $\pi: \mathcal{O}_{X}(1) \rightarrow X$ induces a homotopy equivalence

$$
\pi^{\sharp}: Z_{k}(X) \longrightarrow Z_{k+1}\left(\mathcal{O}_{X}(1)\right) .
$$

In other words, for every non-negative integer $r$ the map $\pi: \mathcal{O}_{X}(1) \rightarrow X$ induces an isomorphism

$$
\left(\pi^{\sharp}\right)^{*}: \pi_{r} Z_{k}(X) \longrightarrow \pi_{r} Z_{k+1}\left(\mathcal{O}_{X}(1)\right) .
$$

In this section we prove the following intersection version of the Lawson Suspension Theorem.

Theorem 3.3. For every pair of non-negative integers $k, r$, every perversity $\bar{q}$, and every perversity $\bar{p}$ so that for every $i \geq 0$ there is an inequality $k-i+1+\bar{p}(i) \geq 0$ the projection map $\pi: \mathcal{O}_{X}(1) \rightarrow X$ induces an isomorphism

$$
I_{\bar{q}} \pi_{r}\left(Z_{k}(X)^{\bar{p}}\right) \longrightarrow I_{\bar{q}} \pi_{r}\left(Z_{k+1}\left(\mathcal{O}_{X}(1)\right)^{\bar{p}}\right),
$$

where $Z_{k+1}\left(\mathcal{O}_{X}(1)\right)^{\bar{p}}$ is a filtration of $Z_{k+1}\left(\mathcal{O}_{X}(1)\right)$ with the following skeleta:

$$
Z_{k+1}\left(\mathcal{O}_{X}(1)\right)^{\bar{p}, 0}=Z_{k+1}\left(\mathcal{O}_{X}(1)\right)
$$

and for $l \geq 1 Z_{k+1}\left(\mathcal{O}_{X}(1)\right)^{\bar{p}, l}$ so that $c \in Z_{k+1}\left(\mathcal{O}_{X}(1)\right)^{\bar{p}, l}$ if there is $j \geq l$ with

$$
\operatorname{dim}\left(|c| \cap \pi^{-1} X_{\text {can }}^{j}\right) \geq k-j+\bar{p}(j)+1 .
$$


Remark. After some minor modifications in the following proof of Theorem 3.3 one can show that for any finite filtration $\mathcal{F}$ of $X$ if $k \geq \bar{t}_{c}-\bar{p}$, then the map $\pi: \mathcal{O}_{X}(1) \rightarrow X$ induces an isomorphism

$$
I_{\bar{q}} \pi_{r}\left(Z_{k}(X), Z_{k}(\mathcal{F})^{\bar{p}}\right) \longrightarrow I_{\bar{q}} \pi_{r}\left(Z_{k+1}\left(\mathcal{O}_{X}(1)\right), Z_{k+1}\left(\pi^{\sharp} \mathcal{F}\right)^{\bar{p}}\right) .
$$

The main part of the proof of Theorem 3.3 is a description of some deformation of compact families of algebraic cycles of bounded degrees. As was pointed to us by Paulo Lima-Filho, the general case can be proved without reference to any group completion or stabilization result, by exploiting the fact that the spaces of algebraic cycles have compactly generated topology (for more details see $[\mathrm{FL}],[\mathrm{LLM}]$ ).

Proof. The map $\pi: \mathcal{O}_{X}(1) \rightarrow X$ induces a homomorphism

$$
\left(\pi^{\sharp}\right)_{*}: I_{\bar{q}} \pi_{r}\left(Z_{k}(X)^{\bar{p}}\right) \longrightarrow I_{\bar{q}} \pi_{r}\left(Z_{k+1}\left(\mathcal{O}_{X}(1)\right)^{\bar{p}}\right),
$$

because for every perversity $\bar{p}$

$$
\pi^{\sharp}\left(Z_{k}(X)^{\bar{p}, l}\right)=\pi^{\sharp}\left(Z_{k}(X)\right) \cap Z_{k+1}\left(\mathcal{O}_{X}(1)\right)^{\bar{p}, l} .
$$

In other words,

$$
\operatorname{dim}\left(|c| \cap X^{j}\right) \geq \operatorname{dim}|c|-j+\bar{p}(j)+1
$$

if and only if

$$
\operatorname{dim}\left(\left|\pi^{\sharp} c\right| \cap \pi^{\sharp} X^{j}\right) \geq \operatorname{dim}\left|\pi^{\sharp} c\right|-j+\bar{p}(j)+1 .
$$

Let $\mathcal{F} \subset \mathcal{C}_{k+1}\left(\mathcal{O}_{X}(1)\right)$ be the monoid of effective $(k+1)$-cycles $c$ of $\mathcal{O}_{X}(1)$ satisfying for every $l \geq 0$ the equality

$$
\operatorname{dim}\left(|c| \cap X^{l}\right)=\operatorname{dim}\left(|c| \cap \pi^{\sharp} X^{l}\right)-1 .
$$

Let $\widetilde{\mathcal{F}}$ be the subgroup of $Z_{k+1}\left(\mathcal{O}_{X}(1)\right)$ generated by $\mathcal{F}$. We equip $\mathcal{F}$ and $\widetilde{\mathcal{F}}$ with the induced by $Z_{k}(X)^{\bar{p}}$ filtrations $\mathcal{F}^{\bar{p}}$ and $\tilde{\mathcal{F}}^{\bar{p}}$ respectively

$$
\begin{aligned}
& \mathcal{F}^{\bar{p}, l}=\mathcal{F} \cap \mathcal{C}_{k}(X)^{\bar{p}, l}, \\
& \widetilde{\mathcal{F}}^{\bar{p}, l}=\widetilde{\mathcal{F}} \cap Z_{k}(X)^{\bar{p}, l} .
\end{aligned}
$$

It is easy to see that the homomorphism

$$
I_{\bar{q}} \pi_{r}\left(Z_{k}(X)^{\bar{p}}\right) \longrightarrow I_{\bar{q}} \pi_{r}\left(Z_{k+1}\left(\mathcal{O}_{X}(1)\right)^{\bar{p}}\right)
$$

factors through $I_{\bar{q}} \pi_{r}\left(\widetilde{\mathcal{F}}^{\bar{p}}\right)$. Thus the proof of Theorem 3.3 can be divided into two parts.

Assertion 1. The composition

$$
Z_{k}(X) \stackrel{\pi^{\sharp}}{\longrightarrow} \pi^{\sharp} Z_{k}(X) \longrightarrow \widetilde{\mathcal{F}}
$$

induces an isomorphism

$$
I_{\bar{q}} \pi_{r}\left(Z_{k}(X)^{\bar{p}}\right) \longrightarrow I_{\bar{q}} \pi_{r}\left(\widetilde{\mathcal{F}}^{\bar{p}}\right) .
$$

Assertion 2. For every perversity $\bar{p}$ so that for each $i \geq 0$ there is an inequality $k-i+1+\bar{p}(i) \geq 0$ the inclusion $\widetilde{\mathcal{F}} \hookrightarrow Z_{k+1}\left(\mathcal{O}_{X}(1)\right)$ induces an isomorphism

$$
I_{\bar{q}} \pi_{r}\left(\widetilde{\mathcal{F}}^{\bar{p}}\right) \longrightarrow I_{\bar{q}} \pi_{r}\left(Z_{k+1}\left(\mathcal{O}_{X}(1)\right)^{\bar{p}}\right) .
$$


It is clear that the map $Z_{k}(X) \stackrel{\pi^{\sharp}}{\longrightarrow} \pi^{\sharp} Z_{k}(X)$ from Assertion 1 induces an isomorphism

$$
I_{\bar{q}} \pi_{r}\left(Z_{k}(X)^{\bar{p}}\right) \longrightarrow I_{\bar{q}} \pi_{r}\left(\pi^{\sharp} Z_{k}(X)^{\bar{p}}\right) .
$$

Thus to prove Assertion 1 one has to show that the inclusion $\pi^{\sharp} Z_{k}(X) \rightarrow \widetilde{\mathcal{F}}$ induces an isomorphism

$$
I_{\bar{q}} \pi_{r}\left(\pi^{\sharp} Z_{k}(X)^{\bar{p}}\right) \longrightarrow I_{\bar{q}} \pi_{r}\left(\widetilde{\mathcal{F}}^{\bar{p}}\right) .
$$

Lawson showed (see [L2]) that there is a deformation retraction $\varphi_{t}: \tilde{\mathcal{F}} \rightarrow$ $\pi^{\sharp} Z_{k}(X)$ of $\tilde{\mathcal{F}}$ onto $\pi^{\sharp} Z_{k}(X)$. It is easy to see that if $X$ and $Y$ are spaces equipped with finite filtrations $X=\left\{X^{l}\right\}$ and $y=\left\{Y^{l}\right\}$ respectively and $f: X \rightarrow Y$ is a homotopy equivalence so that $f\left(X^{l}\right)=Y^{l}$, then $f$ induces an isomorphism of the intersection homotopy groups

$$
I_{\bar{q}} \pi_{r}(X, X) \longrightarrow I_{\bar{q}} \pi_{r}(Y, y)
$$

for every $r \geq 0$ and every perversity $\bar{q}$. Thus, the deformation retraction $\varphi_{t}$ induces an isomorphism on intersection homotopy groups if for every $t \in[0,1]$

$$
\varphi_{t}\left(\tilde{\mathcal{F}}^{\bar{p}, l}\right)=\pi^{\sharp} Z_{k}(X)^{\bar{p}, l} .
$$

The above equality is proved as follows.

The deformation retraction $\varphi_{t}: \tilde{\mathcal{F}} \rightarrow \pi^{\sharp} Z_{k}(X)$ for $t \in[0,1)$ is given by scalar multiplication by $-\ln (1-t)$ in the fibers of $\mathcal{O}_{X}(1)$. On $\widetilde{\mathcal{F}}$ the family $\varphi_{t}$ extends continuously to $t=1$. The map

$$
\varphi_{1}: \widetilde{\mathcal{F}} \longrightarrow \pi^{\sharp}\left(Z_{k}(X)\right)
$$

is the retraction defined by the formula

$$
\varphi_{1}(c)=\pi^{\sharp}(c \cdot X),
$$

where $c \cdot X$ is an algebro-geometric intersection of $c$ with the zero section of $\mathcal{O}_{X}(1)$.

Since for every $c \in \widetilde{\mathcal{F}}$

$$
\operatorname{dim}\left(|c| \cap X^{l}\right)+1=\operatorname{dim}\left(|c| \cap \pi^{\sharp} X^{l}\right)
$$

and

$$
\operatorname{dim}\left(\left|\varphi_{1}(c)\right| \cap \pi^{\sharp} X^{l}\right)=\operatorname{dim}\left(\left|\pi^{\sharp} c\right| \cap \pi^{\sharp} X^{l}\right)=\operatorname{dim}\left(|c| \cap X^{l}\right)+1,
$$

we have the required equality

$$
\varphi_{1}\left(\widetilde{\mathcal{F}} \cap Z_{k+1}\left(\mathcal{O}_{X}(1)\right)^{\bar{p}, l}\right)=\pi^{\sharp}\left(Z_{k}(X)^{\bar{p}, l}\right) .
$$

The proof of Assertion 2 is based on the following lemma whose proof is deferred to the end of this section.

Lemma 3.4. Let $\bar{p}$ be a perversity so that for every $i \geq 0$ there is an inequality $k-i+1+\bar{p}(i) \geq 0$. Then for every compact subset $K$ of the monoid $\mathcal{C}_{k+1}\left(\mathcal{O}_{X}(1)\right)$ there is a constant $d_{0}$ so that for every $d>d_{0}$ there is a continuous family

$$
F_{t}^{d}: \mathcal{C}_{k+1}\left(\mathcal{O}_{X}(1)\right) \longrightarrow \mathcal{C}_{k+1}\left(\mathcal{O}_{X}(1)\right)
$$

of continuous monoid homomorphisms defined for $0 \leq t \leq 1$ with the property that

(A) $F_{0}^{d}$ is the multiplication by $d$.

(B) $F_{t}^{d}(K) \subset \widetilde{\mathcal{F}}$ for all $t>0$. 
(C) For every $t \in[0,1]$ the map $F_{t}$ preserves the filtration $\mathcal{C}_{k+1}\left(\mathcal{O}_{X}(1)\right)^{\bar{p}}$ of $\mathcal{C}_{k+1}\left(\mathcal{O}_{X}(1)\right)$. That is,

$$
F_{t}^{d}\left(K \cap \mathcal{C}_{k+1, r}\left(\mathcal{O}_{X}(1)\right)^{\bar{p}, l}\right)=F_{t}^{d}(K) \cap \mathcal{C}_{k+1, r}\left(\mathcal{O}_{X}(1)\right)^{\bar{p}, l} .
$$

Suppose $f: \mathbb{S}_{r} \rightarrow Z_{k+1}\left(\mathcal{O}_{X}(1)\right)$ is of perversity $\bar{q}$ with respect to the filtration $Z_{k+1}\left(\mathcal{O}_{X}(1)\right)^{\bar{p}}$. There is a non-negative constant $e$ so that $f: \mathbb{S}_{r} \rightarrow Z_{k+1}\left(\mathcal{O}_{X}(1)\right)_{\leq e}$, where $Z_{k+1}\left(\mathcal{O}_{X}(1)\right)_{\leq e}$ is the image of the compact set

$$
\coprod_{r+s \leq e} \mathcal{C}_{k+1, r}\left(\mathcal{O}_{X}(1)\right) \times \mathcal{C}_{k+1, s}\left(\mathcal{O}_{X}(1)\right)
$$

by the quotient map

$$
\mathfrak{C}_{k+1}\left(\mathcal{O}_{X}(1)\right) \times \mathfrak{C}_{k+1}\left(\mathcal{O}_{X}(1)\right) \longrightarrow Z_{k+1}\left(\mathcal{O}_{X}(1)\right) .
$$

Let $K$ from Lemma 3.4 be the sphere $\mathbb{S}_{r}$ and let

$$
F_{t}^{d}: \mathcal{C}_{k+1, r}\left(\mathcal{O}_{X}(1)\right) \longrightarrow \mathcal{C}_{k+1, d r}\left(\mathcal{O}_{X}(1)\right)
$$

be the associated map for some $d \geq d_{0}$. Every $F_{t}^{d}$ induces a map

$$
\Phi_{t}^{d}: Z_{k+1}\left(\mathcal{O}_{X}(1)\right)_{\leq e} \longrightarrow Z_{k+1}\left(\mathcal{O}_{X}(1)\right)_{\leq d e}
$$

given by the formula

$$
\Phi_{t}^{d}\left(c-c^{\prime}\right)=F_{t}^{d}(c)-F_{t}^{d}\left(c^{\prime}\right)
$$

for any pair

$$
\left(c, c^{\prime}\right) \in \coprod_{r+s \leq e} \mathcal{C}_{k+1, r}\left(\mathcal{O}_{X}(1)\right) \times \mathcal{C}_{k+1, s}\left(\mathcal{O}_{X}(1)\right) .
$$

Consider the homotopy

$$
f_{t}=\Phi_{t}^{d+1} \circ f-\Phi_{t}^{d} \circ f .
$$

The condition (A) of Lemma 3.4 implies that $f_{0}=f$. From the condition (B) for every $t>0$ we have $f_{t}(x) \in \widetilde{\mathcal{F}}$ for every $x \in \mathbb{S}_{r}$. The last condition of Lemma 3.4 implies that $\Phi_{t}^{d}$ preserves the filtration $Z_{k+1}\left(\mathcal{O}_{X}(1)\right)^{\bar{p}}$ and hence $f_{t}$ is a map of perversity $\bar{q}$ for an arbitrary $\bar{q}$. This finishes the proof of surjectivity of the map from Assertion 2. The injectivity is proved in the similar way.

The proof of Lemma 3.4. In his fundamental paper [L2] Lawson proved that for every compact subset $K$ of the monoid $\mathcal{C}_{k+1}\left(\mathcal{O}_{X}(1)\right)$ there is a constant $d_{0}$ so that for every $d>d_{0}$ there is a continuous family

$$
F_{t}: \mathcal{C}_{k+1}\left(\mathcal{O}_{X}(1)\right) \longrightarrow \mathcal{C}_{k+1}\left(\mathcal{O}_{X}(1)\right)
$$

of continuous monoid homomorphisms defined for $0 \leq t \leq 1$ with the property that

(A) $F_{0}$ is the multiplication by $d$.

(B) $F_{t}(K)$ is contained in the space of effective $(k+1)$-cycles of $\mathcal{O}_{X}(1)$ intersecting the base $X$ of $\mathcal{O}_{X}(1)$ properly.

We are going to sketch a construction of the family $F_{t}$ and show that it satisfies the conditions of Lemma 3.4 under the assumption that for every $i \geq 0$ there is an inequality $k-i+1+\bar{p}(i) \geq 0$.

Fix a linear embedding $\mathbb{P}_{n+1} \subset \mathbb{P}_{n+2}$ and two points $x_{0}, x_{1} \in \mathbb{P}_{n+2}-\mathbb{P}_{n+1}$. The projections

$$
\pi_{k}: \mathbb{P}_{n+2}-\left\{x_{k}\right\} \longrightarrow \mathbb{P}_{n+1}, \quad k=0,1
$$


give each set $\mathbb{P}_{n+2}-\left\{x_{k}\right\}$ the structure of a holomorphic line bundle over $\mathbb{P}_{n+1}$. Let $\pi_{k}$ be the restriction of $\pi_{k}$ to $\left(\pi_{k}\right)^{-1}\left(\mathcal{O}_{X}(1)\right)$ and let $D$ be an effective divisor of degree $d$ in $\mathbb{P}_{n+2}$ so that $x_{0}, x_{1} \notin|D|$. Any effective cycle $c \in \mathcal{C}_{k+1}\left(\mathcal{O}_{X}(1)\right)$ can be "lifted" to a cycle with support in $D \cap\left(\pi_{0}\right)^{-1}\left(\mathcal{O}_{X}(1)\right)$. This lifting is defined to be the intersection

$$
\Psi_{D}(c)=\left(\pi_{0}\right)^{-1}(c) \cdot D
$$

of the divisor $D$ with the inverse image of $c$ by the projection $\pi_{0}$. This gives us a continuous map

$$
\Psi_{D}: \mathcal{C}_{k+1}\left(\mathcal{O}_{X}(1)\right) \longrightarrow \mathcal{C}_{k+1}\left(\pi_{0}^{-1}\left(\mathcal{O}_{X}(1)\right) \cap \pi_{1}^{-1}\left(\mathcal{O}_{X}(1)\right)\right) .
$$

Consider now the family of divisors $t D, 0 \leq t \leq 1$, given by scalar multiplication by $t$ in the bundle

$$
\pi_{0}:\left(\pi_{0}\right)^{-1}\left(\mathcal{O}_{X}(1)\right) \longrightarrow \mathcal{O}_{X}(1) .
$$

We assume $x_{1} \notin t D$ for all such $t$. The above construction gives us a family of transformations

$$
F_{t} \stackrel{\text { def }}{=}\left(\pi_{1}\right)_{*} \circ \Psi_{t D}: \mathcal{C}_{k+1}\left(\mathcal{O}_{X}(1)\right) \longrightarrow \mathcal{C}_{k+1}\left(\mathcal{O}_{X}(1)\right)
$$

for $0 \leq t \leq 1$ such that $F_{0}$ is the multiplication by $d$.

Using the same arguments as in [L2] it is easy to see that for a fixed cycle $c \in \mathcal{C}_{k+1}\left(\mathcal{O}_{X}(1)\right)$ the set $B_{c}$ of those $D \in \mathcal{C}_{n+1, d}\left(\mathbb{P}_{n+2}\right)$ for which for some $l$ and $t$

$$
F_{t}\left(c \cdot \pi^{\sharp} X^{l}\right) \subset X
$$

is of codimension greater than or equal to

$$
\min _{l}\left\{\left(\begin{array}{c}
\operatorname{dim}\left(c \cdot \pi^{\sharp} X^{l}\right)+d \\
d
\end{array}\right)-1\right\} .
$$

For every $c \in \mathcal{C}_{k+1}\left(\mathcal{O}_{X}(1)\right)^{\bar{p}, l}$ the dimension of the intersection $c \cdot \pi^{\sharp} X^{l}$ is greater than or equal to one, because for each $l \geq 0$ we have $k-l+1+\bar{p}(l) \geq 0$. Thus the codimension of $B_{c}$ in $\mathcal{C}_{n+1, d}\left(\mathbb{P}_{n+2}\right)$ is greater than or equal to $\left(\begin{array}{c}d+1 \\ d\end{array}\right)-1=d$.

Suppose $K$ is a compact subset of $\mathcal{C}_{k+1}\left(\mathcal{O}_{X}(1)\right)$ and let $D$ be an effective divisor which lies in the complement of the union $\bigcup_{c \in K} B_{c}$, which is the set of real codimension greater than or equal to $2(d-\operatorname{dim} K)$ in $\mathcal{C}_{n+1, d}\left(\mathbb{P}_{n+2}\right)$. We set $d_{0}=2 \operatorname{dim} K$. It is clear that $F_{t}$ satisfies the condition (A). The other two properties of $F_{t}$ are proved as follows.

It is easy to see that for all $l$ and $t$

$$
F_{t}\left(c \cdot \pi^{\sharp} X^{l}\right) \subset \pi^{\sharp} X^{l} .
$$

If $D$ is chosen as above, then the inequality

$$
F_{t}\left(c \cdot \pi^{\sharp} X^{l}\right) \subset X
$$

does not hold. Therefore

$$
F_{t}\left(c \cdot \pi^{\sharp} X^{l}\right) \nsubseteq X^{l}
$$

and hence

$\operatorname{dim}\left(\left|F_{t}\left(c \cdot \pi^{\sharp} X^{l}\right)\right| \cap X^{l}\right)=\operatorname{dim}\left(\left|F_{t}\left(c \cdot \pi^{\sharp} X^{l}\right)\right| \cap \pi^{\sharp} X^{l}\right)-1=\operatorname{dim}\left(|c| \cap \pi^{\sharp} X^{l}\right)-1$.

The last equality together with the inclusion (16) implies that

$$
\operatorname{dim}\left(|c| \cap \pi^{\sharp} X^{l}\right)=\operatorname{dim}\left(\left|F_{t}\left(c \cdot \pi^{\sharp} X^{l}\right)\right|\right)=\operatorname{dim}\left(\left|F_{t}(c)\right| \cap \pi^{\sharp} X^{l}\right) .
$$


Thus, $F_{t}$ preserves dimensions of intersections of $c$ with $\pi^{\sharp} X^{l}$, but lowers by one the dimension of intersection of $c$ with $X^{l}$. It is easy to see that the above two properties imply that $F_{t}$ satisfies the conditions (B) and (C) of Lemma 3.4.

\section{Operations in Intersection LaWSON HOMOLOGY}

Friedlander and Mazur defined an operation

$$
\mathbf{s}: \pi_{r} Z_{k}(X) \longrightarrow \pi_{r+2} Z_{k-1}(X),
$$

which is a natural transformation of Lawson homology functor and is compatible with flat pull-back of cycles and localization $[\mathrm{FM}]$. In this section we show that under certain conditions the s-operation lifts to intersection Lawson homology .

Theorem 4.1. Let $X$ be a projective variety. Then for every pair of non-negative integers $k, r$, every perversity $\bar{q}$, and every perversity $\bar{p}$ so that for every $i \geq 0$ we have $k-i+\bar{p}(i) \geq 0$, there is a homomorphism

$$
\mathbf{s}_{\bar{p}, \bar{q}}: I_{\bar{q}} \pi_{r}\left(Z_{k}(X)^{\bar{p}}\right) \longrightarrow I_{\bar{q}} \pi_{r+2}\left(Z_{k-1}(X)^{\bar{p}}\right),
$$

so that the diagram

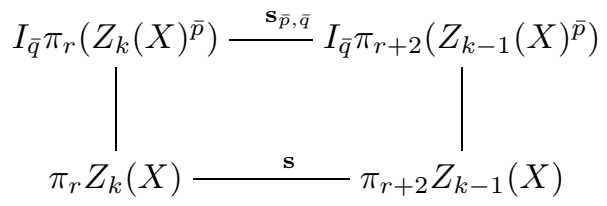

commutes.

Corollary 4.2. The composition of $\mathbf{s}_{\bar{t}_{c}, \bar{q}}$ and $\mathbf{s}$ homomorphisms induces the following commutative diagram:

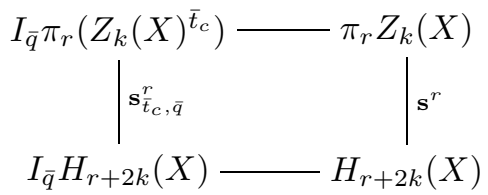

The rest of this section is devoted to the proof of Theorem 4.1 and Corollary 4.2. Let us start from a description of the Friedlander-Mazur s-operation. The s-operation is defined as the composition

$$
\begin{aligned}
\pi_{r} Z_{k}(X) \stackrel{\mathbb{S}_{2} \wedge}{\longrightarrow} \pi_{r+2}\left(\mathbb{S}_{2} \wedge Z_{k}(X)\right) \cong \pi_{r+2}\left(\mathbb{P}_{1} \wedge Z_{k}(X)\right) \stackrel{i_{*} \times i d}{\longrightarrow} \pi_{r+2}\left(Z_{0}\left(\mathbb{P}_{1}\right) \wedge Z_{k}(X)\right) \\
\stackrel{\sharp_{*}}{\longrightarrow} \pi_{r+2}\left(Z_{k+1}\left(\mathbb{P}_{1} \wedge X\right)\right) \cong \pi_{r+2}\left(Z_{k+1}\left(\mathbb{Z}^{2} X\right)\right) \stackrel{\Sigma^{-2}}{\longrightarrow} \pi_{r+2} Z_{k-1}(X),
\end{aligned}
$$

where $\mathbb{S}_{2} \wedge$ is the wedge homomorphism assigning to a map $\mathbb{S}_{r} \longrightarrow Z_{k}(X)$ its wedge

$$
\mathbb{S}_{r+2}=\mathbb{S}_{2} \wedge \mathbb{S}_{r} \longrightarrow \mathbb{S}_{2} \wedge Z_{k}(X)
$$

with the sphere $\mathbb{S}_{2}$, the isomorphism

$$
\pi_{r+2}\left(\mathbb{S}_{2} \wedge Z_{k}(X)\right) \cong \pi_{r+2}\left(\mathbb{P}_{1} \wedge Z_{k}(X)\right)
$$

is induced by an identification of the sphere $\mathbb{S}_{2}$ with the projective line $\mathbb{P}_{1}$, the map $i: \mathbb{P}_{1} \rightarrow Z_{0}\left(\mathbb{P}_{1}\right)$ is the embedding $i(p)=p-p_{\infty}$,

$$
\sharp: Z_{0}\left(\mathbb{P}_{1}\right) \wedge Z_{k}(X) \longrightarrow Z_{k+1}\left(\mathbb{P}_{1} \wedge X\right)
$$


is the complex join map, and

$$
\mathbb{Z}^{-2}: \pi_{r+2}\left(Z_{k}\left(\not^{2} X\right)\right) \longrightarrow \pi_{r+2} Z_{k-1}(X)
$$

is the inverse of the Lawson suspension isomorphism

$$
\not^{2}: \pi_{r+2} Z_{k-1}(X) \longrightarrow \pi_{r+2}\left(Z_{k}\left(\not^{2} X\right)\right)
$$

where $\mathbb{P}_{1}$ is identified with the line in $\mathbb{P}_{n+2} \supset \mathbb{Z}^{2} X$ so that $\mathbb{P}_{1} \sharp X=\mathbb{4}^{2} X$.

Note that $\mathbb{Z}^{2} X-\mathbb{P}_{1}=\mathcal{O}_{\mathcal{O}_{X}(1)}(1)$ and for $k \geq 1$ the inclusion $\mathcal{O}_{\mathcal{O}_{X}(1)}(1) \rightarrow \mathcal{O}_{\nwarrow X X}(1)$ induces an isomorphism

$$
\pi_{r} Z_{k+1}\left(\not \not^{2} X\right) \cong \pi_{r} Z_{k+1}\left(\mathcal{O}_{\mathcal{O}_{X}(1)}(1)\right) .
$$

Hence, the map

$$
\sharp_{*} \circ\left(i_{*} \times i d\right) \circ\left(\mathbb{S}_{2} \wedge(-)\right): \pi_{r} Z_{k}(X) \longrightarrow \pi_{r+2} Z_{k+1}\left(\mathbb{Z}^{2} X\right)
$$

can be identified with a map

$$
\mu: \pi_{r} Z_{k}(X) \longrightarrow \pi_{r+2} Z_{k+1}\left(\mathcal{O}_{\mathcal{O}_{X}(1)}(1)\right) .
$$

It is easy to see that for every pair of perversities $\bar{p}, \bar{q}$ the map $\mu$ lifts to a map

$$
\mu_{\bar{p}, \bar{q}}: I_{\bar{q}} \pi_{r}\left(Z_{k}(X)^{\bar{p}}\right) \longrightarrow I_{\bar{q}} \pi_{r+2}\left(Z_{k+1}\left(\mathcal{O}_{\mathcal{O}_{X}(1)}(1)\right)^{\bar{p}}\right) .
$$

Let $\pi: \mathcal{O}_{X}(1) \rightarrow X$ and $\Pi: \mathcal{O}_{\mp X}(1) \rightarrow \not X$ be the projections. From Theorem 3.3 we know that for $k-1 \geq \bar{t}_{c}-\bar{p}$ the map

$$
\pi^{*}: I_{\bar{q}} \pi_{r+2}\left(Z_{k-1}(X)^{\bar{p}}\right) \longrightarrow I_{\bar{q}} \pi_{r+2}\left(Z_{k}\left(\mathcal{O}_{X}(1)\right)^{\bar{p}}\right)
$$

is an isomorphism. Let us put on $\mathbb{Z X}$ a filtration $\mathcal{F}$ that is obtained from the coarsest Whitney stratification of $\mathcal{O}_{X}(1)$ by adding the vertex of $\mathbb{\&} X$ to every skeleton of $\mathcal{O}_{X}(1)$. As was pointed out in the remark after Theorem 3.3, for $k \geq \bar{t}_{c}-\bar{p}$ the map

$$
\Pi^{*}: I_{\bar{q}} \pi_{r+2}\left(Z_{k}(\not X), \mathcal{F}^{\bar{p}}\right) \longrightarrow I_{\bar{q}} \pi_{r+2}\left(Z_{k+1}\left(\mathcal{O}_{\Sigma X}(1)\right),\left(\Pi^{\sharp}\right)^{\bar{p}}\right)
$$

is an isomorphism. If $k \geq 1$, then the inclusion $\mathcal{O}_{\mathcal{O}_{X}(1)}(1) \rightarrow \mathcal{O}_{\S X}(1)$ induces an isomorphism

$$
I_{\bar{q}} \pi_{r+2}\left(Z_{k+1}\left(\mathcal{O}_{\mathcal{O}_{X}(1)}(1)\right)^{\bar{p}}\right) \longrightarrow I_{\bar{q}} \pi_{r+2}\left(Z_{k+1}\left(\mathcal{O}_{\Sigma X}(1)\right),\left(\Pi^{\sharp}\right)^{\bar{p}}\right) .
$$

Hence, for $k-1 \geq \bar{t}_{c}-\bar{p}$, the composition $\left(\Pi^{*} \circ \pi^{*}\right)^{-1} \circ \mu_{\bar{p}, \bar{q}}$ gives a map

$$
\mathbf{s}_{\bar{p}, \bar{q}}: I_{\bar{q}} \pi_{r}\left(Z_{k}(X)^{\bar{p}}\right) \longrightarrow I_{\bar{q}} \pi_{r+2}\left(Z_{k-1}(X)^{\bar{p}}\right),
$$

which is a lift of the Friedlander-Mazur operation $\mathbf{s}$ to the intersection Lawson homology of $X$. In other words, for $k-1 \geq \bar{t}_{c}-\bar{p}$, there is a commutative diagram

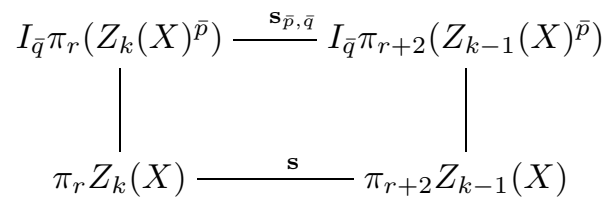

with the vertical arrows the forgetful homomorphisms.

Note that, for every $k \geq 1$, the iteration of $\mathbf{s}_{\bar{p}, \bar{q}}$ gives a map

$$
\mathbf{s}_{\bar{t}_{c}, \bar{q}}^{r}: I_{\bar{q}} \pi_{r}\left(Z_{k}(X)^{\bar{t}_{c}}\right) \longrightarrow I_{\bar{q}} \pi_{r+2 k}\left(Z_{0}(X)^{\bar{t}_{c}}\right),
$$

which is a lift of the map 


$$
\mathbf{s}^{r}: \pi_{r} Z_{k}(X) \longrightarrow \pi_{r+2 k} Z_{0}(X) \cong H_{r+2 k}(X ; \mathbb{Z})
$$

to the intersection Lawson homology of $X$.

The above property implies that the diagram

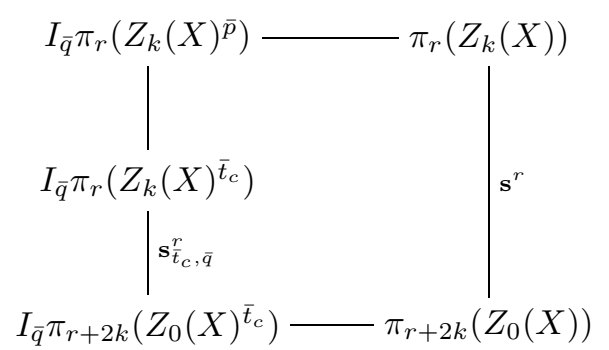

with the unmarked arrows the forgetful homomorphisms, commutes. Hence, the diagram

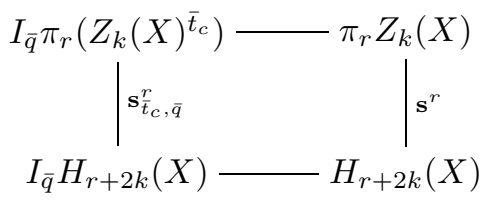

commutes.

\section{REFERENCES}

[Fl] Ch. Flannery, Spaces of algebraic cycles and correspondence homomorphism, to appear in Adv. in Math.

[Fr] E. Friedlander, Algebraic Cycles, Chow Varieties and Lawson Homology, Compositio Math. 77 (1991), 55-93. MR 92a:14005

[FG] E. Friedlander and O. Gabber, Cycle Spaces and Intersection Theory, in Topological Methods in Modern Mathematics, Publish or Perish, 1993. MR 94j:14010

[FL] E. Friedlander and B. Lawson, Moving algebraic cycles of bounded degree, preprint 1995.

[FM] E. Friedlander and B. Mazur, Filtrations on the Homology of Algebraic Varieties, Mem. Amer. Math. Soc., 110 (1994), no. 529. MR 95a:14023

[Gr] P. Gajer, The Intersection Dold-Thom Theorem, Topology 35 (1996), 939-967.

[GM] M. Goresky and R. MacPherson, Intersection Homology Theory, Topology 19 (1980), 135162. MR 82b:57010

[Hi] H. Hironaka, Triangulation of algebraic sets, Proc. Sympos. Pure Math. 29 (1975), 165-185. MR 51:10331

[L1] H. B. Lawson Jr., Spaces of Algebraic Cycles, Surveys in differential geometry, Vol. II (Cambridge, MA, 1993), 137-213, Internat. Press, Cambridge, MA, 1995. CMP 96:08

[L2] Algebraic Cycles and Homotopy Theory, Ann. of Math. (2) 129 (1989), 253-291. MR 90h:14008

[LLM] H. B. Lawson Jr., P. C. Lima-Filho, M.-L. Michelsohn, On Equivariant Algebraic Suspension, Preprint 1995.

[LF1] P. C. Lima-Filho, Lawson Homology for Quasiprojective Varieties, Compositio Math. 84 (1992), 1-23. MR 93j:14007

[LF93] _ Completions and Fibrations for Topological Monoids, Trans. Amer. Math. Soc. 340 (1993), 127-147. MR 94a:55009

[LF2] - The topological group structure of algebraic cycles, Duke Math. J. 75(2) (1994), 467-491. MR 95i:14010

[Lo] S. Lojasiewicz, Ensembles semianalitiques, IHES preprint, 1972.

[Tr] B. Teissier, Variétés polaires II, Algebraic Geometry, Proceedings, La Rábida, Lecture Notes in Math. vol. 961, Springer, 1981, pp. 314-491. MR 85i:32019 
[Wh1] H. Whitney, Local properties of analytic varieties, Differential and Combinatorial Topology, Princeton University Press, 1965, pp. 205-244. MR 32:5924

[Wh2] — , Tangents to an analytic variety, Ann. of Math. (2) 81 (1965), 496-549. MR 33:745

Department of Mathematics, Texas A\&M University, College Station, Texas 77843

Current address: Department of Mathematics, Johns Hopkins University, Baltimore, Maryland 21218 